NBER WORKING PAPER SERIES

\title{
OVERCONFIDENCE, SUBJECTIVE PERCEPTION AND PRICING BEHAVIOR
}

\author{
Pierpaolo Benigno \\ Anastasios G. Karantounias \\ Working Paper 11922 \\ http://www.nber.org/papers/w11922
}

\author{
NATIONAL BUREAU OF ECONOMIC RESEARCH \\ 1050 Massachusetts Avenue \\ Cambridge, MA 02138 \\ January 2006
}

We are grateful to Mike Woodford for useful discussions and comments and to seminar participants at Columbia University, Federal Reserve Board of Governors and NYU. The views expressed herein are those of the author(s) and do not necessarily reflect the views of the National Bureau of Economic Research.

(C2006 by Pierpaolo Benigno and Anastasios G. Karantounias. All rights reserved. Short sections of text, not to exceed two paragraphs, may be quoted without explicit permission provided that full credit, including (C) notice, is given to the source. 
Overconfidence, Subjective Perception and Pricing Behavior

Pierpaolo Benigno and Anastasios G. Karantounias

NBER Working Paper No. 11922

January 2006, Revised March 2006

JEL No. D4, D8, E3

\begin{abstract}
$\underline{\text { ABSTRACT }}$
We study the implications of a particular form of irrationality on the pricing behavior of firms in a monopolistic-competitive market with incomplete information. We assume that firms are overconfident, meaning that they over-estimate their abilities to understand the correct model of the economy. However, we allow firms to obtain information by paying a fixed cost. We find two important implications: i) overconfident firms are less inclined to acquire information; ii) prices might exhibit excess volatility driven by non-fundamental disturbances. We use our model to match some facts related to recent empirical evidence on disaggregated price data for the US economy.

Pierpaolo Benigno

Department of Economics

New York University

269 Mercer Street

New York, NY 10003

and NBER

pierpaolo.benigno@nyu.edu

Anastasios G. Karantounias

Department of Economics

New York University

269 Mercer Street

New York, NY 10003

anastasios.karantounias@nyu.edu
\end{abstract}


Using micro data on consumer prices for the U.S. economy, Klenow and Kryvtsov (2004), hereinafter KK, have documented that prices change frequently with an average absolute size of $8.5 \%$ on a monthly basis and that these frequent movements are equally likely to be positive or negative in sign. In contrast, aggregate inflation averages just at $0.6 \%$ over an horizon of 3 months. This evidence is an important challenge for modelers of inflation dynamics, especially for those who believe that nominal disturbances can have real and highly persistent effects on output. Indeed, Golosov and Lucas (2004) have been able to match this empirical evidence with a model in which pricing decisions are state dependent but subject to a cost of changing prices. In their model the observed high volatility of prices is explained by changes driven by highly volatile idiosyncratic productivity shocks. But, no matter what the source and the size of the other disturbances in the economy, the pricing decisions are dominated by the occurrence of this idiosyncratic component. As a consequence, a nominal disturbance has negligible real effects on output since it mixes up with the idiosyncratic shocks. To counteract this result, Gertler and Leahy (2005) have shown that even a model with state-dependent pricing can be consistent with important real effects of nominal disturbances.

In a recent work, Woodford (2002) has renewed interest on an alternative theory of the real effects of monetary policy that originates from the Phelps (1970) and Lucas (1972) hypothesis of decisionmakers that have imperfect information on the nature of the disturbances that affect the economy. The important acumen of his theory is to observe that it does not really matter whether information is publicly available or not but what matters is the limited ability of agents to process available information, as in the rational inattention theory of Sims (2003). The limited processing ability leads to a "gap between reality and perception" (Woodford, 2002, p.31), which can be modelled as if agents receive noisy signals about the shocks of the economy. In particular, the hidden process of the stochastic disturbance represents the objective "reality", whereas the signals represent the "subjective perception" of the decisionmakers (price-setters) and the "gap between reality and perception" is the difference between the two.

When there is a "gap between reality and perception" and this gap is idiosyncratic to each decisionmaker, then an important source of uncertainty is the "perception" of others together with all their higher-order beliefs on them. The slow adjustment of higher-order beliefs can produce real effects of a nominal shock 
that are highly persistent. The attractive feature of this theory is that it can justify unequal adjustments of prices to different disturbances for the fact that decisionmakers can be more or less attentive to different disturbances. Along this line of reasoning, Maćkowiak and Wiederholt (2005) have been able to match the abovementioned empirical evidence in a model in which price setters have to dichotomize their attention between an idiosyncratic productivity shock and an aggregate nominal shock. If the idiosyncratic disturbance is relatively more volatile, then they pay more attention to it and therefore prices react more to these disturbances. On the other side, when a nominal and less volatile disturbance perturbs the economy, decisionmakers show rational inattention, which combined with the slow adjustment of higher-order beliefs can produce highly persistent effects on output.

In the Woodford-Sims theory the "gap between reality and perception" is the same whether one looks from the "reality" or the "perception" side of the world. Decisionmakers have limited ability to process information but they are rational in all other aspects. This assumption ignores the fact that decisionmakers may exhibit various biases about their subjective perceptions.

We instead depart from a fully rational model. Our aim here is to explain the excess volatility of prices documented by KK with price movements that follow idiosyncratic non-fundamental noises in contrast with the other contributions described above. ${ }^{1}$

In the Woodford-Sims theory, we assume that the "gap between reality and perception" is smaller when one looks from the "subjective mind" of the decisionmaker than from the objective "reality". In practice, this translates into a model in which price setters over-estimate their ability to understand "reality" by overestimating the precision of their signals. This is overconfidence.

That overconfidence accords with "reality" is well documented by psychological studies. For example, in a simultaneous study of US and Swedish drivers, $88 \%$ of the US group and $77 \%$ of the Swedish group asked believed that they are safer drivers than the median (Svenson, 1981), or $90 \%$ of 168 federal magistrate

\footnotetext{
${ }^{1} \mathrm{~A}$ documented example is the following. Frozen concentrated orange juice in the US is for the greatest part produced in Orlando, Florida. The most relevant fundamental variable that changes on a day-to-day basis is then the weather around Orlando. Roll (1984) has shown that weather surprises explain only a small fraction of the observed variability in futures prices and on top of this no other demand or supply factors can be identified to explain more than a small part of it.
} 
judges thought that they are above average as far as their reversal rate on appeal is concerned (Guthrie, Rachlinski and Wistrich, 2001). ${ }^{2}$ Experimental studies (see Soll and Klayman, 2004) have shown that subjective confidence intervals are systematically too narrow given the accuracy of one's information. ${ }^{3}$

A model with irrational behavior gives some degrees of freedom. In order to bound it, we allow our price setters to pay a fixed cost in order to become completely aware of "reality". 4 But who are going to be stuck with their own perception of the world if not the ones that are overconfident and believe that the "gap between reality and perception" is small? In consequence in our model a large fraction of price setters is composed by inattentive and irrational decisionmakers. Woodford's theory then predicts that a nominal disturbance can have large and persistent real effects. ${ }^{5}$ At the same time, the fact that the "gap between reality and perception" is smaller in the "subjective mind" of the decisionmakers than in "reality" helps to explain the high volatility of prices in an unrelated way to fundamental disturbances. Indeed, overconfident decisionmakers place a lot of weight on their subjective perceptions which include the under-estimated nonfundamental noise.

We calibrate our model to be consistent with the mean and the variance of price changes in the KK data. Moreover, we tight our parametrization to be compatible with the persistence of the effects of nominal disturbances on output as found in the VAR literature.

With rational price setters, our model implies that the average absolute size of price changes is of the order of only $2 \%$. But with overconfidence it increases up to the value found in the data. In particular, for a reasonable degree of overconfidence documented in experimental studies we find that the average absolute size of price

\footnotetext{
${ }^{2}$ See for example Compte and Postlewaite (2004) for further references.

${ }^{3}$ In finance researchers have analyzed the implications of overconfidence on the financial markets, see Daniel, Hirshleifer and Subrahmanyam (2001), Daniel, Hirshleifer, Teoh (2002) and Scheinkman and Xiong (2003) among others.

${ }^{4} \mathrm{~A}$ recent related work is that of Reis (2005) in which he shows that information costs can rationalize a model where fully rational price setters have different information sets as in Mankiw and Reis (2002). However in his model information flows with delays while in our model, as in the Sims's theory, information flows in each period with a noisy channel.

${ }^{5}$ In a recent work Morris and Shin (2006) have emphasized that in models with forwardlooking expectations even the existence of a small fraction of uninformed agents about the future path of fundamentals can generate persistence in the price behavior.
} 
changes is of the order of $4-5 \%$. This is quite a success for a model that does not rely at all on idiosyncratic fundamental shocks.

The paper is structured as follows. Section 1 presents the static model under full information. Section 2 discusses the incomplete-information model. Section 3 approximates and solves the incomplete-information model. Section 4 discusses the price implications of the static model and the role of overconfidence. Section 5 extends the model to an infinite horizon. Section 6 performs the calibration of the model and studies the success of the model in explaining the empirical evidence. Section 7 presents some statistical tests to study whether overconfidence is statistically detectable. Section 8 concludes.

\section{Model}

In this section, we present a partial-equilibrium model of price-setting behavior in which firms have full information on the structure, parameters and variables of interest. ${ }^{6}$ We consider a continuum of firms indexed by $i$ on the unit interval $[0,1]$. Each firm produces a good that is differentiated in the preferences of consumers. We do not explicitly model neither consumer preferences nor their optimization problem. We just assume what is needed to characterize the pricesetting problem of firms. Firms are profit maximizers and set their prices in a monopolistic-competitive market. The problem of a generic firm $j$ is to choose the price of its product $P(j)$ to maximize real profits given by

$$
\frac{P(j)}{P} Y(j)-\frac{W}{P} L(j)
$$

where $Y(j)$ is the demand of good $j$ given by

$$
Y(j)=\left(\frac{P(j)}{P}\right)^{-\varepsilon} Y
$$

that depends on the relative price of the good $j$ with respect to the general price index $P$ given by

$$
P=\left[\int_{0}^{1} P(i)^{1-\varepsilon} d i\right]^{\frac{1}{1-\varepsilon}}
$$

\footnotetext{
${ }^{6}$ The model is similar to the one used in Ball and Romer $(1989,1991)$ and Blanchard and Kiyotaki (1987).
} 
and on aggregate production $Y^{7}$ The parameter $\varepsilon$ (with $\varepsilon>1$ ) denotes the elasticity of substitution across differentiated goods in consumer preferences. Firms use labor $L(j)$ to produce goods through the production function $Y(j)=A L(j)$, where $A$ is a productivity shock common to all firms; $W$ is the nominal wage paid for one unit of labor in the labor market.

In this market (not modelled here) we assume that the labor-supply schedule implies the following relation between real wage and aggregate production

$$
\frac{W}{P}=Y^{\eta}
$$

with $\eta>0 .{ }^{8}$ We assume the existence of a monetary authority that has a perfect control on the level of nominal spending in the economy. It follows that

$$
M=P Y
$$

where $M$, which may be labelled as money supply, is indeed controlled by the monetary authority.

We can substitute (1.2), (1.4) and (1.5) into (1.1) to define a profit function of firm $j$ as

$$
\pi(P(j), P, \theta) \equiv\left[\frac{P(j)}{P}-\frac{1}{A}\left(\frac{M}{P}\right)^{\eta}\right]\left(\frac{P(j)}{P}\right)^{-\varepsilon}\left(\frac{M}{P}\right) .
$$

Profits of firm $j$ are a function of the action (in game-theoretic sense) of firm $j$, $P(j)$, of the actions of all other firms synthesized by the index $P$ and the vector $\theta \equiv(A, M)$. Firm $j$ is of measure zero with respect to the aggregate, so its pricing decision does not affect the general price index $P$. We denote with $P^{\dagger}(j)$ the optimal price choice of firm $j$ given by

$$
P^{\dagger}(j)=\frac{\varepsilon}{\varepsilon-1} \frac{P}{A}\left(\frac{M}{P}\right)^{\eta}
$$

\footnotetext{
${ }^{7}$ In (1.3), we use the index $i$ to emphasize that firm $j$ is small with respect to the overall market.

${ }^{8}$ This labor-supply schedule can be derived from the optimizing-behavior of households in a general-equilibrium model. In particular, $\eta$ would be a combination of the risk-aversion coefficient in consumer preferences and of the Frisch elasticity of substitution of labor supply, or in case of local labor market of $\varepsilon$ as well. Assuming a more general labor-supply schedule does not change the following analysis.
} 
which is just the familiar markup rule over marginal cost. Since the right-hand side of (1.7) is independent of $j$, all firms set the same price. It follows that $P^{\dagger}(j)=P=P^{*}$ for all $j$ where

$$
P^{*}=\left(\frac{\varepsilon}{\varepsilon-1} \frac{1}{A}\right)^{\frac{1}{\eta}} M .
$$

For later use, we can use the definition of $P^{*}$ to write (1.7) as

$$
P^{\dagger}(j)=P^{1-\eta} P^{* \eta}
$$

This rewriting shows that the parameter $\eta$ determines whether price-setting decisions are strategic complement (the case $0<\eta<1$ ) or strategic substitutes $(\eta>1)$. In the strategic-complement case, there is a positive elasticity between the individual optimal price and the aggregate price level. The elasticity is negative when pricing decisions are strategic substitutes.

\section{Incomplete information}

In this section, we formalize the incomplete-information version of the above model. First, we assume that firms do not know the realization of the vector $\theta$. In particular we assume that $\theta$ belongs to a set of possible outcomes $\Theta$ and that each firm knows that there is a probability distribution $f(\theta)$ over the possible outcomes. ${ }^{9}$ Each firm can observe a signal $s^{j}$ that belongs to a set $S$. We model incomplete information by assuming that each firm can only observe its own signal, which is then private information.

Each firm believes that the signals are related to the possible outcomes $\theta$ through a likelihood function $\tilde{l}^{j}\left(s^{j} \mid \theta\right)$ which might be specific to the firm $j$ but common knowledge to all firms. We call $\tilde{l}^{j}\left(s^{j} \mid \theta\right)$ the subjective likelihood function to distinguish it from the objective likelihood $l^{j}\left(s^{j} \mid \theta\right)$ that characterizes the true conditional probability distribution of signals. In this way we depart from full rationality. ${ }^{10}$

\footnotetext{
${ }^{9} \mathrm{We}$ are assuming that $\Theta$ is finite for the sake of the discussion.

${ }^{10}$ In the literature on ambiguity in the priors, see Gilboa and Schmeidler (1989), it is assumed instead that there can be multiple likelihoods, whereas we assume a single likelihood, which may not be the correct one though.
} 
Given its private signal $s^{j}$, each firm can update its probability distribution over the set of outcomes $\Theta$ using Bayes' rule and obtain the posterior distribution

$$
\tilde{f}^{j}\left(\theta \mid s^{j}\right)=\frac{\tilde{l}^{j}\left(s^{j} \mid \theta\right) f(\theta)}{\sum_{\Theta} \tilde{l}^{j}\left(s^{j} \mid \theta\right) f(\theta)},
$$

for each $\theta \in \Theta$. However the realization of $\theta$ is not the only element of uncertainty in the profit function (1.6). Indeed profits depend also on the non-observable general price index $P$, which consists of the non-observable prices chosen by other firms and through them on the private signals of the other firms. Since the likelihoods and priors are all common knowledge, each firm can apply rules of conditional probability to obtain the conditional probability distribution of other firms' signals given the realization of its own signal. But this is not enough to characterize all the relevant uncertainty since pricing decisions of other firms depend on their beliefs about the signals of others as well as on the beliefs about others' beliefs and so on ad infinitum. As in a Bayesian game of incomplete information, each firm is not only characterized by its private signal, but also by all the full hierarchy of beliefs about others.

To define the objective function of firms in this incomplete-information game, we need to specify a broader set of states of nature $\Omega$ whose element $\omega$ represents the knowledge and the interactive beliefs of each firm. ${ }^{11}$ Each firm is then characterized by a type $t^{j}$ belonging to a set $T^{j}$ and $t^{j}$ is related to $\omega$ through a function $\tau(\cdot)$ such that $t^{j}=\tau^{j}(\omega)$.

Given this abstract formalization of the states of nature, each firm strategy can be understood as a mapping of the form $P(j)=P\left(t^{j}\right)$ from the space of types to the space of possible prices. Each firm can form a probability distribution $p\left(\omega \mid t^{j}\right)$ over the broader set of states of nature $\Omega$ conditional on being of type $t^{j}$. We can now write the objective function of a generic firm $j$ as

$$
E^{j}\{\pi(P(j), P, \theta)\} \equiv \sum_{\left\{\omega:\left(\tilde{t}^{-j}, \tilde{\theta}\right)=\left(t^{-j}, \theta\right)\right\}} p^{j}\left(\omega \mid t^{j}\right) \pi\left(P(j), P\left(t^{-j}\right), \theta\right),
$$

where $t^{-j}$ denotes all the other types of firms excluding firm $j$ and with $P\left(t^{-j}\right)$ we denote

\footnotetext{
${ }^{11}$ We follow Battigalli (2003) in this construction.
}

$$
P\left(t^{-j}\right)=\left[\int_{0}^{1} P\left(t^{i}\right)^{1-\varepsilon} d i\right]^{\frac{1}{1-\varepsilon}}
$$


In a Bayesian equilibrium a firm of type $t^{j}$ chooses its action $P(j)$ to maximize the objective function (2.9) given the strategy of other firms. We denote this choice by $\tilde{P}(j)$ and it satisfies

$$
\tilde{P}(j)=\frac{E^{j}\left\{P^{\dagger}(j) Z\right\}}{E^{j}\{Z\}},
$$

where $Z$ is defined as $Z \equiv M P^{\varepsilon-2}$ and $P^{\dagger}(j)$ is given by (1.8). In particular, $P^{\dagger}(j)$ denotes the complete-information price. Indeed this is the price that a generic firm would set if it knew the realization of $\omega$. But this price does not necessarily coincide with the full-information price since it is not yet specified whether other firms know $\omega$. According to (2.10), when there is incomplete information, a generic firm $j$ sets its price as a subjective expectation (appropriately weighted) of the price that would be set under complete information.

We enrich the above framework by allowing each firm to acquire complete information on the state of the economy -i.e. to know the state $\omega$. This process of acquisition of information is costly: real profits are reduced by a fixed cost $\tilde{c}^{j}$. Moreover, this decision is conditional on the firm of being of type $t^{j}$. In what follows we label as 'informed' firms the ones that acquire information, while the others are denoted as 'uninformed'.

We have to specify the strategy of each firm in this modified problem. We can compactly write the strategy as a mapping $\sigma(\cdot)$ that maps from the space $T^{j}$ of types to the actions. It now involves two actions: the decision of whether or not to acquire information and the price chosen following this decision. Note that when informed a firm would set its price to $P^{\dagger}(j)$. The expected profit function of a firm $j$ conditional of being of type $t^{j}$ is now

$$
E^{j}\{\pi(P(j), P, \theta)\} \equiv \sum_{\left\{\omega:\left(\tilde{t}^{-j}, \tilde{\theta}\right)=\left(t^{-j}, \theta\right)\right\}} p^{j}\left(\omega \mid t^{j}\right) \pi\left(P(j), \sigma\left(t^{-j}\right), \theta\right) .
$$

A generic firm $j$ of type $t^{j}$ chooses to acquire complete information when the expected increase in profits in doing this is higher than the cost $\tilde{c}^{j}$

$$
E^{j}\left\{\pi\left(P^{\dagger}(j), P, \theta\right)-\pi(\tilde{P}(j), P, \theta)\right\} \geq \tilde{c}^{j}
$$

Indeed since the profit function is concave in the price $P(j)$ then setting $P(j)$ with a finer knowledge of the state of nature does not worsen utility, so that the LHS of the above expression is always non-negative. Having observed the realization of 
its own signal, a firm $j$ evaluates the LHS of (2.11) and acquires information and sets the price $P^{\dagger}(j)$, if (2.11) holds, otherwise it chooses $\tilde{P}(j)$.

\section{Approximation to the incomplete-information model}

In this section we solve the previous model in a log-linear approximation around a steady-state in which $\theta=\bar{\theta}$. As shown in the Appendix, a second-order approximation to the objective function (2.11) leads to a criterion of the form

$$
\operatorname{var}^{j}\left\{p^{\dagger}(j)\right\} \geq c^{j}
$$

where $\operatorname{var}^{j}\{\cdot\}$ denotes the variance operator conditional on the subjective information set, while $p^{\dagger}(j)$ is the log of the price that firm $j$ would set with complete information and $c^{j}$ is a reparametrization of the cost $\tilde{c}^{j}$. The decision of acquiring or not information depends on whether the subjective variance of the price that a firm sets under complete information is higher than the cost $c^{j}$.

An important implication of the approximation taken is that (3.12) can be evaluated using just a log-linear approximation to the equilibrium conditions. In this log-linear approximation, equation (2.10) implies that the log of the price under incomplete information is the expected value of the $\log$ of the price under complete information

$$
\tilde{p}(j)=E^{j} p^{\dagger}(j)
$$

where lower-case letters denote log of the respective variables. ${ }^{12}$ Moreover $p^{\dagger}(j)$ is independent of $j$ and in a log-linear approximation to (1.8) is given by

$$
p^{\dagger}=(1-\eta) p+\eta p^{*}
$$

where $p^{*}$ is the log of the full-information equilibrium price level and $p$ is given by

$$
p=\int_{0}^{1} p(i) d i
$$

as a result of a first-order approximation of (1.3).

\footnotetext{
${ }^{12}$ In the steady-state all firms set the same constant price.
} 
Let us denote with $\mu$ the fraction of firms that in equilibrium decide to keep the subjective information set and assuming without loss of generality that agents $j \in[0, \mu]$ are the ones who remain uninformed, we can write the above equation as

$$
p=\mu \tilde{p}+(1-\mu) p^{\dagger},
$$

where we have defined with $\tilde{p}$ the average price of the subjectively-informed firms as

$$
\tilde{p} \equiv \frac{1}{\mu} \int_{0}^{\mu} \tilde{p}(i) d i
$$

We can then plug (3.15) into (3.14) to obtain

$$
p^{\dagger}=\delta p^{*}+(1-\delta) \tilde{p}
$$

where

$$
\delta \equiv \frac{\eta}{\eta+(1-\eta) \mu}
$$

with $\delta$ a decreasing function of $\mu$ and $\eta \leq \delta \leq 1$ in case of strategic complements $(\eta<1)$ and $\delta$ an increasing function of $\mu$ with $1 \leq \delta \leq \eta$ in case of strategic substitutes $(\eta>1)$.

The set of equations (3.13), one for each firm that remains uninformed, together with (3.16) and (3.17) determine the equilibrium prices of informed and uninformed firms in a first-order approximation to the equilibrium conditions.

To solve for the equilibrium prices, we describe in details the information structure. First, we note that uncertainty about the vector $\theta$ has collapsed to uncertainty about the full-information price $p^{*}$. We assume that $p^{*}$ is a random variable of the form

$$
p^{*}=\bar{p}^{*}+u,
$$

where $\bar{p}^{*}$ is a constant and $u$ is a Gaussian white-noise process with variance $\sigma_{u}^{2}$. It follows that the prior distribution of $p^{*}$ is Gaussian with mean $\bar{p}^{*}$ and variance $\sigma_{u}^{2}$. These priors are common knowledge and correspond to the objective probability distribution of $p^{*}{ }^{13}$ Each firm receives a private signal $s^{j}$ that is linearly related to $p^{*}$ as

$$
s^{j}=p^{*}+\xi^{j},
$$

\footnotetext{
${ }^{13}$ In the non-linear model, these priors correspond to the assumption on the distribution $f(\theta)$.
} 
where $\xi^{j}$ is an idiosyncratic Gaussian noise with mean zero and variance $\bar{\sigma}_{\xi}^{2}$ for each $j$. Moreover $\xi^{j}$ is statistically independent of $u$ as well as of $\xi^{i}$ for each $i \neq j$. All this information is common knowledge. Instead, the realization of the signal $s^{j}$ is private information.

In the previous section, we assumed that the subjective likelihood function $\tilde{l}\left(s_{t}^{j} \mid \theta_{t}\right)$ can be different from the true likelihood through which signals are extracted. We capture this by assuming that the belief, $\bar{\sigma}_{\xi}^{2}$, on the variance of the noise $\xi^{j}$ does not correspond to the true variance $\sigma_{\xi}^{2}$.

Given this information structure, each firm can form its own expectation of the full-information price $p^{*}$ as in a standard signal-extraction problem

$$
E^{j} p^{*}=\left(1-\bar{r}^{j}\right) \bar{p}^{*}+\bar{r}^{j} s^{j}
$$

where the weight $\bar{r}^{j}$ is defined as

$$
\bar{r}^{j}=\bar{r} \equiv \frac{1}{1+\bar{\lambda}}
$$

with

$$
\bar{\lambda}=\frac{\bar{\sigma}_{\xi}^{2}}{\sigma_{u}^{2}} .
$$

Since $\bar{\lambda}$ is common and common knowledge across the different firms, then $\bar{r}^{j}$ is independent of $j$ and equal to a common $\bar{r}$. In particular $\bar{\lambda}$ represents the noise-tofundamental variance ratio and can be interpreted as an index of confidence in how a firm's private signal is a good representation of the full-information price. Lower values of $\bar{\lambda}$ implies a higher weight to the signal when firms form expectations of the full-information price and then a high degree of confidence on the subjective information set. Since $\sigma_{\xi}^{2}$ does not necessarily correspond to the prior $\bar{\sigma}_{\xi}^{2}$, we define a 'true' degree of confidence $\lambda$ as

$$
\lambda=\frac{\sigma_{\xi}^{2}}{\sigma_{u}^{2}},
$$

with a respective value for the weight $r=1 /(1+\lambda)$. When $\bar{\lambda}<\lambda(\bar{r}>r)$ firms are over-confident and trust more their subjective perception of the world than in the case they would have known the true likelihood. In the opposite case, there is under-confidence. 
Each firm forms its own expectation of the signals of others as

$$
E^{j} s^{i}=E^{j} p^{*}=(1-\bar{r}) \bar{p}^{*}+\bar{r} s^{j}
$$

which is then a first-order expectation belief. ${ }^{14}$ Furthermore, each firm can form its subjective expectation of others' first-order expectation belief as

$$
E^{j} E^{i} s^{k}=E^{j}\left[(1-\bar{r}) \bar{p}^{*}+\bar{r} s^{i}\right]=(1-\bar{r}) \bar{p}^{*}+\bar{r} E^{j} p^{*}
$$

and so on. We can construct all the hierarchy of expectation beliefs, first and higher-order expectations as well as all the other relevant moments that identify a generic type $t^{j}$ in the first-order approximation to the model.

To solve for the equilibrium prices of informed and uninformed agents and for the equilibrium fraction of firms that acquire or not information, we first guess that $\mu$ is known to each type $t^{j}$. We then verify that this is indeed the case. Given this guess, we can substitute (3.17) into (3.13) to get

$$
\tilde{p}(j)=\delta E^{j} p_{t}^{*}+(1-\delta) E^{j} \tilde{p}
$$

which can be averaged across all uninformed price setters to obtain

$$
\tilde{p}=\delta \bar{E} p^{*}+(1-\delta) \bar{E} \tilde{p}
$$

where we have defined the operator $\bar{E}(\cdot) \equiv \frac{1}{\mu} \int_{0}^{\mu} \bar{E}(\cdot) d i$ which represents the average expectation among the uninformed firms. We can iterate the above expression to obtain

$$
\tilde{p}=\delta \sum_{k=0}^{\infty}(1-\delta)^{k} \bar{E}^{(k+1)} p^{*}
$$

where the $(k+1)$-order average expectation operator is defined as $\bar{E}^{(k+1)}(\cdot) \equiv$ $\bar{E}\left(\bar{E}^{(k)}(\cdot)\right)$ for each $k \geq 1 .{ }^{15}$ It follows that the average price of uninformed firms is a linear combination of their higher-order average expectations of the full-information price. We can plug (3.20) into (3.19) to obtain

$$
\tilde{p}(j)=\delta E^{j} p^{*}+\delta(1-\delta) \sum_{k=0}^{\infty}(1-\delta)^{k} E^{j} \bar{E}^{(k+1)} p^{*} .
$$

\footnotetext{
${ }^{14}$ See Allen et al. (2005) and Amato and Shin (2003, 2006) for examples of solutions of problems with iterated expectations.

${ }^{15}$ Under the restriction that $\eta(2 \mu-1)<2 \mu, \delta$ is such that $|1-\delta|<1$.
} 
The price set by an uninformed firm depends on its own expectation of the fullinformation price and its own expectation of the average expectation (of uninformed firms) of the full-information prices as well as on all higher-order expectations. Since (3.18) holds for all uninformed firms, we can obtain that

$$
\bar{E} p^{*}=(1-\bar{r}) \bar{p}^{*}+\bar{r} p^{*}
$$

where we have implicitly assumed that the law of large number holds on a positive measure $^{16}$

$$
\frac{1}{\mu} \int_{0}^{\mu} \xi^{i} d i=0
$$

It follows that the firm $i$ 's expectation of the average expectation of the fullinformation price is given by

$$
\begin{aligned}
E^{i} \bar{E} p^{*} & =(1-\bar{r}) \bar{p}^{*}+r E^{i} p^{*} \\
& =(1-\bar{r}) \bar{p}^{*}+\bar{r}(1-\bar{r}) \bar{p}+\bar{r}^{2} s^{i}
\end{aligned}
$$

from which it follows that the second-order average estimate is given

$$
\bar{E}^{(2)} p^{*}=(1-\bar{r})(1+\bar{r}) \bar{p}^{*}+\bar{r}^{2} p^{*}
$$

By re-iterating the above arguments, we get that the k-fold average expectation of the full-information price is

$$
\bar{E}^{k} p^{*}=\left(1-\bar{r}^{k}\right) \bar{p}^{*}+\bar{r}^{k} p^{*}
$$

We can substitute (3.23) into (3.20) to obtain

$$
\begin{aligned}
\tilde{p} & =\frac{1-\bar{r}}{1-(1-\delta) \bar{r}} \bar{p}^{*}+\frac{\bar{r} \delta}{1-(1-\delta) \bar{r}} p^{*} \\
& =\frac{\eta(1-\bar{r})+(1-\eta)(1-\bar{r}) \mu}{\eta+(1-\eta)(1-\bar{r}) \mu} \bar{p}^{*}+\frac{\bar{r} \eta}{\eta+(1-\eta)(1-\bar{r}) \mu} p^{*} .
\end{aligned}
$$

Substituting (3.24) into (3.14) we obtain that the price set by any informed firm is

$$
p^{\dagger}=\frac{(1-\bar{r})(1-\eta) \mu}{\eta+(1-\eta)(1-\bar{r}) \mu} \bar{p}^{*}+\frac{\eta}{\eta+(1-\eta)(1-\bar{r}) \mu} p^{*} .
$$

\footnotetext{
${ }^{16}$ See Uhlig (1996) for the conditions under which this holds.
} 
Given (3.25), it follows that a generic firm $j$ decides to acquire information if the following inequality holds

$$
\left[\frac{\eta}{\eta+(1-\eta)(1-\bar{r}) \mu}\right]^{2} \operatorname{var}^{j}\left\{p^{*}\right\} \geq c^{j},
$$

where $\operatorname{var}^{j}\left\{p_{t}^{*}\right\}$ is the variance of the full-information price level conditional on the subjective information set of type $t^{j}$. This is given by

$$
\operatorname{var}^{j}\left\{p^{*}\right\}=\sigma_{u}^{2}\left(1-\bar{r}^{j}\right)
$$

We can then write the above inequality as

$$
\left[\frac{\eta}{\eta+(1-\eta)(1-\bar{r}) \mu}\right]^{2} \sigma_{u}^{2}\left(1-\bar{r}^{j}\right) \geq c^{j}
$$

where we have kept the distinction - since it matters for the discussion that followsbetween the own degree of confidence $\bar{r}^{j}$ and the others' degree of confidence $\bar{r}$ although we have assumed that they are the same. ${ }^{17}$

According to (3.26), several parameters of the model drive the incentives for firm $j$ to acquire information. The higher is the prior on the variance of the full-information price, $\sigma_{u}^{2}$, the higher are the incentives to acquire information. Obviously, the lower the $\operatorname{cost} c^{j}$, the higher those incentives. In the case of strategic complementarity in the pricing decision, $0<\eta<1$, the higher is the fraction of firms that are acquiring information (i.e. the lower the $\mu$ ) the higher are the incentives for the individual firm to acquire information. This result is of the same nature as the one found by Ball and Romer (1989) in a similar model but with only imperfect information, in which firms's decisions are on whether to change or not prices.

Each firm's decision is also influenced by the degree of confidence in the informativeness of the signal. If $\bar{r}^{j}$ is high $\left(\bar{\lambda}^{j}\right.$ is low $)$, i.e. it believes that the signal conveys good information on the full-information price, it will not have incentives to acquire finer information. A high degree of confidence implies that firms are going to be stuck with their perceptions of the world when setting their prices.

Interestingly, if the confidence of others increases ( $\bar{\lambda}$ decreases and $\bar{r}$ increases) then the price under complete information has higher subjective variance since the

\footnotetext{
${ }^{17}$ Indeed, (3.25) could have been derived even if we didn't have the same $\bar{r}^{i}$ by defining $\bar{r}$ $\equiv\left(\int_{0}^{\mu} \bar{r}^{i} d i\right) / \mu$ and by assuming a law of large numbers to hold for $\int_{0}^{\mu} \xi^{i} \bar{r}^{i} d i=0$.
} 
average price of uninformed firms is getting close to the full information price, as shown in (3.21). Then, each individual firm has higher incentives to acquire information and imitate other firms -when pricing decisions are strategic complements.

We move to characterize the equilibrium value of $\mu$, under the assumption $\bar{r}^{j}=\bar{r}$ for each $j$. We define

$$
c^{*} \equiv\left[\frac{\eta(1-\bar{r})^{\frac{1}{2}}}{\eta+(1-\eta)(1-\bar{r}) \mu}\right]^{2} \sigma_{u}^{2}
$$

and note that (3.26) implies that all firms with $c^{j}$ less than $c^{*}$ acquire information. Assuming that the distribution of $c^{j}$ is common knowledge with a density function $f\left(c^{j}\right)$ on a support $[\underline{\mathrm{c}}, \bar{c}]$, then the measure $\mu$ of agents that remain uninformed is given by

$$
\int_{c^{*}}^{\bar{c}} f(c) d c=\mu .
$$

This solution confirms our initial guess that $\mu$ is a function of known parameters and then known to each type $t^{j}$. The properties of the distribution function $f(\cdot)$ determines the existence and the characteristics of the equilibrium. Indeed when $c^{j}=c$ for each $j$, multiple equilibria are possible for the same reasons as they occur in the imperfect-information model of Ball and Romer (1989). For other distributions $f(\cdot)$ multiple equilibria might disappear. Since this is not the focus of this work, we assume that $f(\cdot)$ is such that there exists a unique equilibrium.

Using Leibniz rule and noting that $c^{*}=c^{*}(\bar{r}, \mu(\bar{r}))$, we obtain that $(3.27)$ implies

$$
\frac{d \mu}{d \bar{r}}=-\frac{f\left(c^{*}\right) \frac{\partial c^{*}}{\partial \bar{r}}}{1+f\left(c^{*}\right) \frac{\partial c^{*}}{\partial \mu}}
$$

The denominator of (3.28) is positive when $\mu$ is a stable fixed point of the LHS of (3.27). ${ }^{18}$ Moreover

$$
\frac{d c^{*}}{d \bar{r}}=\frac{\eta^{2} \sigma_{u}^{2}}{[\eta+(1-\eta)(1-\bar{r}) \mu]^{3}}[-\eta+(1-\bar{r})(1-\eta) \mu]
$$

is negative for all $\eta$ such that $\eta>\bar{\eta}$ where $\bar{\eta}$ is a function of $\bar{r}$ bounded above by $1 / 2$ and decreasing to zero when $\bar{r}$ increases to one. It follows that for values of $\eta$

\footnotetext{
${ }^{18}$ The solution $\mu$ that we refer as stable is the one in which the LHS of (3.27), considered as a function of $\mu$, intersects the 45 degrees line with a positive slope less than unitary.
} 
in this range an increase in the degree of confidence $(\bar{\lambda}$ decreases and $\bar{r}$ increases $)$ raises the fraction of firms that remain uninformed.

In particular, overconfidence increases the equilibrium fraction of firms that choose not to acquire information in comparison to the equilibrium fraction of rational signal-extraction problem.

\section{Price implications of incomplete information and overconfidence}

In the previous section, we have shown how to determine the fraction of firms that in equilibrium decide to remain uninformed. A high degree of confidence under standard regularity conditions implies a higher fraction of uninformed firms. In this section we study the price implications of the model and in particular the relation between excess volatility of prices and overconfidence.

A first important implication is that the model displays two levels of heterogeneity: at a first stage there are differences in prices between informed and uninformed firms, second, within uninformed firms, prices are related to the realization of subjective signals. We can rewrite equation (3.25) and show that the price of the informed firms is

$$
p^{\dagger}=\bar{p}^{*}+(1+\bar{\lambda}) \hat{k} u,
$$

where

$$
\hat{k}=\frac{\bar{r} \eta}{\eta+(1-\eta)(1-\bar{r}) \mu} .
$$

The prices of the informed firms react only to the fundamental shocks of the model. On the opposite the uninformed firms set their prices as a subjective expectation of $p^{\dagger}$, based on their signals which include also non-fundamental noises. We obtain that a generic uninformed firm $j$ sets

$$
\tilde{p}(j)=\bar{p}^{*}+\hat{k} u+\hat{k} \xi^{j},
$$

while the average price of uninformed firms is given by

$$
\tilde{p}=\bar{p}^{*}+\hat{k} u .
$$


We first discuss how prices react to fundamental shocks. Following (4.29) prices of informed firms react less than proportionally to fundamental shocks when pricing decisions are strategic complements, since $(1+\bar{\lambda}) \hat{k}<1$, but more than proportionally in the strategic-substitute case. As shown in (4.30), the response of uninformed firms is always smaller than that of informed firms, since $\bar{\lambda}>0$.

However, prices of uninformed firms react also to non-fundamental shocks, $\xi^{j}$, in the same proportion as they do with fundamental shocks.

Overconfidence - modelled as the possibility that uninformed firms misinterpret the model of the economy by using an incorrect likelihood - can affect the volatility of prices. Using equation (4.30), we obtain that the "true" variance of prices for a generic uninformed agent $j$ is

$$
\operatorname{var}\{\tilde{p}(j)\}=(1+\lambda) \hat{k}^{2} \sigma_{u}^{2} .
$$

Equation (4.29) implies that the variance of the prices of informed firms is given by

$$
\operatorname{var}\left\{p^{\dagger}\right\}=(1+\bar{\lambda})^{2} \hat{k}^{2} \sigma_{u}^{2}
$$

It follows that the ratio of the volatilities of prices of uninformed and informed firms is given by

$$
\frac{\operatorname{var}\{\tilde{p}(j)\}}{\operatorname{var}\left\{p^{\dagger}\right\}}=\left[\frac{(1+\lambda)^{\frac{1}{2}}}{(1+\bar{\lambda})}\right]^{2} .
$$

In a model in which there is no discrepancy between the "perception" and "reality" so that the signal-extraction problem is rational (i.e. $\lambda=\bar{\lambda}$ ), prices of uninformed firms are always less volatile than informed firms. With overconfident firms, it is instead possible for the reverse to happen. It is sufficient that $(1+\bar{\lambda})<(1+\lambda)^{\frac{1}{2}}$. In particular it is required to have that the true volatility of the idiosyncratic noise $\sigma_{\xi}^{2}$ is high enough with respect to the perceived $\bar{\sigma}_{\xi}^{2}$.

A second important implication of overconfidence is that it is even possible to have excess volatility of the price of an individual uninformed firm with respect to the full-information (fundamental) price. Indeed we obtain that

$$
\frac{\operatorname{var}\{\tilde{p}(j)\}}{\operatorname{var}\left\{p^{*}\right\}}=\left[\frac{\zeta(1+\lambda)^{\frac{1}{2}}}{(1+\bar{\lambda})}\right]^{2}
$$

where $\zeta$ is a positive parameter given by $\zeta=\hat{k} / \bar{r}$ such that $\zeta<1(\zeta>1)$ when pricing decisions are strategic complements (substitutes). To have excess volatility 
of the prices of uninformed firms with respect to fundamentals, it is required that $(1+\bar{\lambda})<\zeta(1+\lambda)^{\frac{1}{2}}$ which is then a more (less) stringent condition than before when pricing decisions are strategic complements (substitutes). ${ }^{19}$

Overconfidence has two important roles in this model. On one side, it implies that a higher fraction of firms is going to decide optimally not to acquire information and just pay attention to their own perceptions. On the other side, the prices of individual uninformed firms can be more volatile than fundamental disturbances and this volatility can be mainly driven by the noise in the perception of the full-information price. These two results prepare the stage for our explanation of the high volatility of individual prices which is found in the data without neither assuming high volatility of fundamental disturbances nor sacrificing persistence in the response of prices to fundamental shocks-among which monetary shocks. Indeed, in the dynamic extension of the above model, the fact that overconfident price setters are less prone to acquire information implies that there can be a high proportion of this kind of subjectively-driven price setters. Woodford (2002) has shown that in this dynamic model higher-order expectations matter for determining persistent effects of output and prices following exactly those disturbances to which agents are subjectively informed. On the other side, the existence of subjectively-informed firms which are overconfident and have irrational beliefs can produce in this context an excess volatility of prices with respect to fundamentals.

\section{Infinite-horizon model}

In this section, we consider an extension of the previous model to an infinite horizon. We assume that each firm does not know the realization of the sequence $\left\{\theta_{t}\right\}_{t=t_{0}}^{\infty}$. However, each firm has a prior distribution on the sequence $\left\{\theta_{t}\right\}_{t=t_{0}}^{\infty}$ that coincides with the correct distribution and which is common knowledge. In each period and contingency, each firm can observe a private signal $s_{t}^{j}$. In particular the sequence of signals $\left\{s_{t}^{j}\right\}_{t=t_{0}}^{\infty}$, one for each $j$, is related to the sequence $\left\{\theta_{t}\right\}_{t=t_{0}}^{\infty}$ through a likelihood function which is known and common knowledge but, as

\footnotetext{
${ }^{19}$ Note that with no overconfidence $(\lambda=\bar{\lambda})$ the ratio is always smaller that unity even in the case of strategic substitutes $(\zeta>1)$. This is clear if we note that $\hat{k}=\delta /(\delta+\lambda)$ and that the ratio is less than unity when $Q(\delta)=\lambda \delta^{2}-2 \lambda \delta-\lambda^{2}<0$, which holds for the permissible $\delta$, i.e. such that $|1-\delta|<1$.
} 
before, does not necessarily coincide with the correct likelihood function. As in the previous model, incomplete information is modelled by assuming that each firm knows only its own realization of the signals and not those of the others, as well as not the price index and the individual prices. Each firm has the option to acquire information on the states of nature - in the abstract sense as it applies to the dynamic extension of the previous model. This can be done by paying a cost $\tilde{c}^{j}$ which is known. Once the cost is paid the firm remains in the 'informed' state forever, otherwise it continues to observe only its private signal and each period decides whether to acquire or not information.

We assume that firms choose prices to maximize the expected discounted value of profits given by

$$
E_{t_{0}}^{j} \sum_{t=t_{0}}^{\infty} \beta^{t-t_{0}} \pi\left(P_{t}(j), P_{t}, \theta_{t}\right),
$$

where $\beta$ is such that $0<\beta<1 .^{20} E_{t_{0}}^{j}$ is the appropriate expectation operator conditional on the information at time $t_{0}$. Prices are set freely in each period. An 'informed' firm sets its price as

$$
P_{t}^{\dagger}(j)=P_{t}^{1-\eta} P_{t}^{* \eta}
$$

for each period $t$ after having paid the information cost. An 'uninformed' firm instead sets its price as

$$
\tilde{P}_{t}(j)=\frac{E_{t}^{j}\left\{P_{t}^{\dagger}(j) Z_{t}\right\}}{E_{t}^{j}\left\{Z_{t}\right\}},
$$

where $Z_{t}$ has the same definition as before and the expectation operator is conditional on the type $t_{t}^{j}$ that a firm $j$ has at time $t$.

To characterize the decision for a generic firm $j$ to acquire or not information, we guess an equilibrium and then verify that prices and information decisions are consistent with that equilibrium. The analysis is simplified by noting that the fraction of firms that remain uninformed each period cannot increase over time, i.e. $\left\{\mu_{t}\right\}_{t=t_{0}}^{+\infty}$ is a non-increasing sequence. Of the many equilibria that can exist, we are interested in ones in which $\mu_{t}=\mu$ for each $t \geq t_{0}$. In particular, in these stationary equilibria, whichever firm decides to be informed does it in the first period. For this to be optimal, the strategy of getting information in the first period should

\footnotetext{
${ }^{20} \mathrm{We}$ can generalize the analysis that follows by assuming a stochastic discount factor to evaluate real profits across contingencies and time.
} 
give higher expected discounted profits than the strategy of waiting until a generic time $T$, given the equilibrium strategies of all other firms. In particular at time $t_{0}$ the expected profits to acquire immediately information and pay the cost should be higher than the strategy of remaining with the subjective information until a generic period $T$ and pay the cost in that period. For a generic firm $j$ to become informed at time $t_{0}$, the following inequality should hold for each $T>t_{0}$

$$
E_{t_{0}}^{j} \sum_{t=t_{0}}^{T-1} \beta^{t-t_{0}} \pi\left(\tilde{P}_{t}(j), P_{t}, \theta_{t}\right)-\beta^{T} \tilde{c}^{j} \leq E_{t_{0}}^{j} \sum_{t=t_{0}}^{T-1} \beta^{t-t_{0}} \pi\left(P_{t}^{\dagger}(j), P_{t}, \theta_{t}\right)-\tilde{c}^{j},
$$

which can be rewritten

$$
E_{t_{0}}^{j} \sum_{t=t_{0}}^{T-1} \beta^{t-t_{0}}\left\{E_{t}^{j}\left[\pi\left(P_{t}^{\dagger}(j), P_{t}, \theta_{t}\right)-\pi\left(\tilde{P}_{t}(j), P_{t}, \theta_{t}\right)\right]\right\} \geq \tilde{c}^{j}\left(1-\beta^{T}\right) .
$$

We take a second-order approximation of the above problem around a stationary point with unitary relative prices to obtain

$$
E_{t_{0}}^{j} \sum_{t=t_{0}}^{T-1} \beta^{t-t_{0}} \operatorname{var}_{t}^{j}\left\{p_{t}^{\dagger}\right\} \geq c^{j}\left(1-\beta^{T}\right)
$$

We guess, and verify later, that in the equilibrium $\operatorname{var}_{t}^{j}\left\{p_{t}^{\dagger}\right\}$ is a constant that does not depend on $j$ and is also independent of $t$ in a stationary filtering problem. The above condition then simplifies to

$$
\operatorname{var}_{t}^{j}\left\{p_{t}^{\dagger}\right\} \geq c^{j}(1-\beta)
$$

which is also independent of $T$. It is then also easy to check that the condition (5.32) with the reverse inequality is all that is needed to verify, in a second-order approximation, that for a generic firm $j$ it is always optimal to remain uninformed. Then (5.32) for each firm $j$ determines the equilibrium fraction of firms that remain uninformed in equilibrium. We verify now that $\operatorname{var}_{t}\left\{p_{t}^{\dagger}\right\}$ is constant and that $\mu$ is also a constant and known within the information set of each type of firm at time $t_{0}$. As before, we just need to characterize the equilibrium values of prices in a log-linear approximation to the equilibrium.

It is still true that the set of equations (3.13), one for each firm that remains uninformed, together with (3.16) and (3.17) determine the equilibrium prices of 
informed and uninformed firms in a first-order approximation to the equilibrium conditions. We continue to assume that each firm receives a private signal $s_{t}^{j}$ that is related linearly to $p_{t}^{*}$ as

$$
s_{t}^{j}=p_{t}^{*}+\xi_{t}^{j}
$$

where $\xi_{t}^{j}$ is an idiosyncratic Gaussian noise with mean zero and variance $\sigma_{\xi}^{2}$ for each $j$. We assume that $\xi_{t}^{j}$, for each $j$, is statistically independent of the sequence $\left\{p_{t}^{*}\right\}$ as well as of the sequence $\left\{\xi_{t}^{i}\right\}$ for each $i \neq j$. All this information is common knowledge, but the realization of the private signal $s_{t}^{j}$ is private information. As before, we assume that the variance of the noises $\bar{\sigma}_{\xi}^{2}$ does not correspond to the true variance $\sigma_{\xi}^{2}$. We allow now $\left\{p_{t}^{*}\right\}$ to be a first-order autoregressive stochastic process of the form

$$
p_{t}^{*}=\bar{p}^{*}+\rho p_{t-1}^{*}+u_{t}
$$

with $|\rho| \leq 1$ where $u_{t}$ is Gaussian noise with mean zero and variance $\sigma_{u}^{2}$. The assumption of persistence of the unobservable shock can in principle be a source of complication in the solution of the model, for an infinite dimensional state might be necessary to keep track of all the beliefs and higher-order beliefs of other firms. Woodford (2002) has shown that the dimension of the hidden-state space is finite in the same model as the one presented here but with all firms assumed to be uninformed and no endogenous decision of acquiring information. Details of the solution can be found in the appendix.

In this solution, the general price index evolves according to

$$
p_{t}=\bar{p}^{*}+\rho(1-\hat{k}) p_{t-1}+\rho \hat{k} p_{t-1}^{*}+[\delta(1-\mu)(1-\hat{k})+\hat{k}] u_{t},
$$

where $\hat{k}$ is a parameter explained in the appendix. ${ }^{21}$ We obtain that the price of informed firms follows

$$
p_{t}^{\dagger}=\bar{p}^{*}+\rho(1-\hat{k}) p_{t-1}^{\dagger}+\rho \hat{k} p_{t-1}^{*}+[\delta(1-\hat{k})+\hat{k}] u_{t}
$$

while the price of uninformed firms follows

$$
\tilde{p}_{t}(j)=\bar{p}^{*}+\rho(1-\hat{k}) \tilde{p}_{t-1}(j)+\rho \hat{k} p_{t-1}^{*}+\hat{k}\left(u_{t}+\xi_{t}^{j}\right) .
$$

${ }^{21}$ The $\hat{k}$ in the dynamic model is a different function of other parameters than the $\hat{k}$ in the static model. We use the same notation, since when $\rho=0$ the two expressions coincide. 
As in the static model, $\tilde{p}_{t}(j)=E_{t}^{j} p_{t}^{\dagger}$ so that subtracting (5.36) from (5.35) we can obtain the evolution of the contemporaneous forecast error

$$
p_{t}^{\dagger}-E_{t}^{j} p_{t}^{\dagger}=\rho(1-\hat{k})\left(p_{t-1}^{\dagger}-E_{t-1}^{j} p_{t-1}^{\dagger}\right)+\delta(1-\hat{k}) u_{t}-\hat{k} \xi_{t}^{j}
$$

from which it follows that the contemporaneous variance of $p_{t}^{\dagger}$ in a stationary solution is given by

$$
\operatorname{var}_{t}^{j}\left\{p_{t}^{\dagger}\right\}=\frac{1+\bar{\lambda}\left[1-\rho^{2}(1-\hat{k})\right]^{2}}{\left[1-\rho^{2}(1-\hat{k})^{2}\right]} \bar{\lambda} \hat{k}^{2} \sigma_{u}^{2}
$$

where the static model is nested under the assumption that $\rho=0$. We can now evaluate (5.32). It follows that equilibrium fraction of uninformed firms is implicitly defined by the same condition as (3.27) where now instead $c^{*}$ is given by

$$
c^{*} \equiv \frac{1+\bar{\lambda}\left[1-\rho^{2}(1-\hat{k})\right]^{2}}{\left[1-\rho^{2}(1-\hat{k})^{2}\right]} \frac{\bar{\lambda} \hat{k}^{2} \sigma_{u}^{2}}{(1-\beta)} .
$$

The main qualitative results of the static model hold in this extension with some qualifications. Indeed it is still the case that overconfidence is needed for the volatility of prices of uninformed to be higher than that of informed. Indeed we show in the appendix that the ratio of the unconditional variances between informed and uninformed firms is higher than the unitary value, i.e.

$$
\frac{\operatorname{var}\left\{\tilde{p}_{t}(i)\right\}}{\operatorname{var}\left\{p_{t}^{\dagger}\right\}}>1
$$

if and only if

$$
\lambda>2 \bar{\lambda}+\bar{\lambda}^{2}\left[1-\rho^{2}(1-\hat{k})^{2}\right],
$$

which nests the previous result. In this dynamic model, it does not only matter the difference between the 'true' and the subjective degree of confidence, but also other parameters. Indeed since $\hat{k}<1$, the discrepancy between $\lambda$ and $\bar{\lambda}$ that is needed in order to have excessive volatility of the uninformed prices is smaller than in the static case. The reason is that the persistence of the shock process makes past estimates useful to forecast the future evolution of the state. But this leads to a larger reliance on their subjective perception (signals) and therefore, comparatively to the static case, agents are driven more by their perceptions. As a consequence, the amount of overconfidence needed to have excess volatility is less. This is also the case if the mass of uninformed agents $(\mu)$ increases since $\hat{k}$ becomes smaller and if the degree of strategic complementarity increases, i.e. $\eta$ becomes smaller. 


\section{How much overconfidence is needed to match the absolute size of individual price changes?}

In this section, we aim at evaluating the empirical performance of the above model. The objective is to be able to explain in a reasonable way the large absolute price changes that KK found in the data. With reasonable we mean that our model should be also consistent with some other moments of the data reported in their study and at the same time require information costs which are in line with other empirical studies. Moreover, we want to show that our explanation can be consistent with a model that allows for a persistent response of output to a nominal spending disturbance. ${ }^{22}$

Considered all together, these objectives are quite ambitious for a model that has only one fundamental shock and does not make any use of fundamental idiosyncratic disturbances. The only source of heterogeneity are the private signals of the firms. In our model the only possibilities for increasing the size of the absolute price changes are to increase either the degree of overconfidence in the signals or the variance of the fundamental disturbance. By including other types of idiosyncratic shocks we can in principle relax the amount of overconfidence needed to explain the data and then be more successful, but at the cost of obscuring the message of the previous model.

Moreover, there can be several other possible mechanisms through which prices change that we have not analyzed in the previous model - again for the sake of simplicity and tractability. Most important is the fact that indeed Bils and Klenow (2004) found that prices are sticky with a median duration of 4.3 months. But in our model firms change their price in each period, even if they have incomplete information. Because of this negligence, we decide to measure the time period of our model in quarters, during which we might reasonably assume that all the firms had the time to adjust their prices. It is reasonable to think that the large absolute price changes that KK document on a monthly basis can be smaller at quarterly frequencies. This again would be an argument in our favor, since it is going to require a lower degree of overconfidence.

The parameters of the model are $\left(\rho, \bar{p}^{*}, \sigma_{u}^{2}, \bar{\lambda}, \frac{\lambda}{\lambda}, \eta, \varepsilon, F\right)$, where $F$ indicates the

\footnotetext{
${ }^{22}$ For calibration purposes we are going to treat the shock in the full infomation price as a nominal spending shock.
} 
parameters that characterize the distribution of the costs. We calibrate $\rho=1$ in a way that our model can be consistent with a unit root in the general price index, which is in general not rejected by the data. ${ }^{23}$ Under this assumption equilibrium output in deviation from the steady state (i.e. $y_{t} \equiv \ln Y_{t} / \bar{Y}$ ) follows the process

$$
y_{t}=(1-\hat{k}) y_{t-1}+(1-c) u_{t}
$$

where $c=(\delta \mu+1-\delta) \hat{k}+\delta(1-\mu)$ and $\delta=\eta /(\eta+(1-\eta) \mu)$. From this process we can deduce that the half-life of the response of output to a nominal spending shock is given by $\tau=-\ln 2 / \ln (1-\hat{k})$. Since we want our model to display a persistent response of output and at the same time be consistent with all the other facts, we assume that $\tau$ is equal to 4 quarters as it is suggested by the VAR literature and which is a reasonable value according to the discussion of Woodford (2003, ch. $3)$. This assumption implies that $\hat{k}=0.159$. Under the assumption of $\rho=1$, the process of the inflation rate is given $b^{24}$

$$
\pi_{t}=\hat{k} \bar{p}^{*}+(1-\hat{k}) \pi_{t-1}+c u_{t}-(c-\hat{k}) u_{t-1} .
$$

In their sample, KK find an average monthly percentage price change (excluding sales) among firms that change prices of $1.11 \%$ with a standard deviation of $1.14 \%$. This corresponds to a calibration of $\bar{p}^{*}=1.11$ for the mean price change among all firms in our model. Furthermore, their data on the variance of the inflation provide an additional moment condition to match for the variance of the model inflation rate, $\sigma_{\pi}^{2}$, which according to $(6.40)$ is just a function of the form

$$
\sigma_{\pi}^{2}=f\left(\mu, \hat{k}, \eta, \sigma_{u}^{2}\right)
$$

pinning down a relation between $\eta, \sigma_{u}^{2}$ and $\mu$ for given $\hat{k}, \sigma_{\pi}^{2}$.

Recent studies on the costs of price adjustment like Zbaracki et al. (2003) have shown that managerial and customer costs of price adjustment constitute a large fraction of firms profits. In fact, managerial costs (which refer to information gathering, decision-making and communicating-to-sales-team costs) are $4.61 \%$ of the

\footnotetext{
${ }^{23}$ We have experimented with data on the non-shelter CPI for the sample period of KK (1988:1-2003:4) and found that a null of a unit root in the price level cannot be rejected.

${ }^{24}$ Note that since the idiosyncratic noise washes out in the aggregate, the amount of overconfidence doesn't affect the aggregate dynamics of inflation and the relevant mean and standard deviation.
} 
profits. We use this evidence to measure the costs of acquiring information in our model. We parameterize parsimoniously the distribution of costs per period as a fraction of steady state profits by assuming a uniform distribution with a minimum cost of zero and a mean cost of $4.61 \% .{ }^{25}$ Furthermore, we assume an elasticity of substitution between the differentiated products $\varepsilon=6$, which corresponds to a markup under full information of $20 \%$. Given these assumptions, and noting that $\bar{\lambda}=\delta(1-\hat{k}) / \hat{k}^{2}$ equations (3.27) and (5.38) imply the following relation

$$
g\left(\mu, \hat{k}, \eta, \sigma_{u}^{2}\right)=0
$$

which is then another implicit relation between $\eta, \sigma_{u}^{2}$ and $\mu$ for given $\hat{k}$.

The parameter $\eta$ is critical for determining whether pricing decisions are strategic complements or substitutes and plays a crucial role in determining the persistence of the response of output to a monetary shock, as discussed in Woodford (2003, ch. 3). Indeed, Woodford (2003, ch. 3) has shown that when a sufficient degree of strategic complementarity is assumed, i.e. a low value of $\eta$ around 0.15 , then a sticky-price model can account for a prolonged response of output. On the opposite case, Chari et al. (2000) assume a value of $\eta$ equal to 2.25, leading to pricing decisions that are strategic complements and then have argued that a sticky-price model is not able to generate enough persistence. In this work, we take an agnostic view on $\eta$ and experiment how our results may differ for a range of values for this parameter. In particular, we report results for a range of $\eta$ that goes from the low number assumed in Woodford (2003, ch. 3) to the high number used in Chari et al. (2000). Having fixed $\eta$, then equations (6.41) and (6.42) determine $\mu$ and $\sigma_{u}^{2}$. We can determine $\delta$ and, bearing in mind that $\bar{\lambda}=\delta(1-\hat{k}) / \hat{k}^{2}$, we can also determine the degree of confidence compatible with our calibration strategy.

Table 1 presents the results of the calibration and in particular how the parameters and the equilibrium fraction $\mu$ are influenced by the chosen value of $\eta$. We observe that lower values of $\eta$ require higher variance of the fundamental shock $\sigma_{u}^{2}$ to match the variance of price changes in the data and at the same time have the model consistent with a half-life of 4 quarters and reasonable information costs. However, the degree of confidence on the signals, which is $\bar{\lambda}$, that measures how good the signals are as a proxy of the fundamental shock, is low for low values of $\eta$. This perhaps indicates that the model is more reasonable when $\eta$ is in this range

\footnotetext{
${ }^{25}$ See details in the appendix.
} 
and a sufficient degree of price complementarity is assumed. Using $\eta=0.15$ we obtain that $83 \%$ of firms maintain a subjective information set when setting their prices. This value decreases as $\eta$ increases and reaches $57 \%$ when $\eta=2.25$.

\section{Table 1: Calibration and Equilibrium Fraction $\mu$}

\begin{tabular}{cclccccc} 
& $\eta=0.15$ & $\eta=0.5$ & $\eta=0.9$ & $\eta=1.1$ & $\eta=1.5$ & $\eta=1.9$ & $\eta=2.25$ \\
\hline $\bar{p}^{*}$ & 1.11 & 1.11 & 1.11 & 1.11 & 1.11 & 1.11 & 1.11 \\
$\sigma_{u}$ & 4.234 & 2.97 & 2.310 & 2.131 & 1.903 & 1.764 & 1.678 \\
$\bar{\lambda}$ & 5.81 & 20.6 & 31.20 & 35.05 & 41.07 & 45.53 & 48.62 \\
$\mu$ & 0.831 & 0.611 & 0.580 & 0.577 & 0.573 & 0.571 & 0.570 \\
\hline
\end{tabular}

We then proceed to simulate the model in order to characterize the pricing behavior of 20000 firms over the sample 1988:1-2003:4 as in KK. Over this sample, we compute the same statistics that they report and in particular we focus on the average over the sample and across firms of the absolute of the changes in the individual prices for two subsequent observations. This statistic corresponds to the statistic $|d p|$ for regular prices of all items in their Table 1. By construction, our simulations are in line with the statistics that they report on the mean and variance of the price changes. ${ }^{26}$

Our results on the variable $|d p|$ depend on the degree of overconfidence assumed. We have defined overconfidence as the ratio $\lambda / \bar{\lambda}$ which is equivalent to $\sigma_{\xi}^{2} / \bar{\sigma}_{\xi}^{2}$, the ratio of the true variance of the noise with respect to the perceived variance. We choose as an index of overconfidence the parameter $\gamma=\sigma_{\xi} / \bar{\sigma}_{\xi}$ which then gives a measure on how much the true standard deviation of the noise exceeds the believed one. We repeat our simulations for the chosen values of $\eta$ by letting $\gamma$ varies from 1 to 8 . In particular $\gamma=1$ corresponds to the rational signal extraction problem. The results are presented in Table 2 .

\footnotetext{
${ }^{26}$ We have repeated the described simulation 1000 times to smooth out any influence of the small sample on the results and we average the statistics across these repeated simulations.
} 
Table 2: Average absolute value of price changes (\%) and overconfidence

\begin{tabular}{c|ccccccc} 
& $\eta=0.15$ & $\eta=0.5$ & $\eta=0.9$ & $\eta=1.1$ & $\eta=1.5$ & $\eta=1.9$ & $\eta=2.25$ \\
\hline$\gamma=1$ & 1.83 & 2.012 & 1.99 & 1.97 & 1.95 & 1.94 & 1.93 \\
$\gamma=2$ & 2.75 & 3.02 & 2.89 & 2.86 & 2.8 & 2.76 & 2.73 \\
$\gamma=3$ & 3.80 & 4.05 & 3.86 & 3.79 & 3.69 & 3.63 & 3.59 \\
$\gamma=4$ & 4.88 & 5.13 & 4.83 & 4.73 & 4.6 & 4.51 & 4.46 \\
$\gamma=5$ & 5.98 & 6.21 & 5.81 & 5.68 & 5.52 & 5.40 & 5.33 \\
$\gamma=6$ & 7.07 & 7.28 & 6.79 & 6.65 & 6.43 & 6.30 & 6.21 \\
$\gamma=7$ & 8.18 & 8.37 & 7.77 & 7.60 & 7.36 & 7.19 & 7.08 \\
$\gamma=8$ & 9.30 & 9.45 & 8.76 & 8.56 & 8.28 & 8.08 & 7.96
\end{tabular}

Table 2 shows for each pair $\gamma$ and $\eta$ the average absolute value of price changes $(|d p|)$ implied by this model. The reference value is the $8.5 \%$ of KK. In a rational signal extraction problem $(\gamma=1)$ we obtain a value close to $2 \%$. But to match their reported value we need a degree of overconfidence close to 7 or 8 , which can be considered as a large number. Indeed, experimental studies, like Soll and Klayman (2004), have shown that on a series of questions where individuals are asked to form an $80 \%$ confidence interval the actual hit rate is around $40 \%$, which can be translated in $\gamma$ being approximately equal to $2.5{ }^{27}$ In more complicated tasks, as forecasting the level of the exchange rate with a confidence interval of 90\% (see Oberlechner and Osler, 2004), the hit rate ranges from 5\% to $70 \%$ with an average of $40 \%$, rationalizing values of $\gamma$ higher than 3. In general values from 2 to 4 can be considered as reasonable.

If we set our target less ambitiously and ask what is the implied $|d p|$ for a degree of overconfidence close to experimental evidence, we find that this can range from $3 \%$ to $5 \%$, doubling the value under a fully rational model and being close to explain more than the $50-60 \%$ of the value found in the data. ${ }^{28}$ We think that these results can be suggestive of the fact that the mechanism we underline can

\footnotetext{
${ }^{27}$ This value can be obtained by rough computation on confidence intervals for normal distributions.

${ }^{28}$ As Table 1 shows, this result does not depend on the value of $\eta$ assumed.
} 
be important to explain the excess volatility of prices -although we acknowledge that there can be other important mechanisms from which we have abstracted in this analysis.

\section{Detecting overconfidence}

One important objection against the above structure is that it is unrealistic to think that firms can only observe their own signals. In reality, firms can gather detailed information on the various components of their profits through which they might be able to infer the structure of the economy and uncover the process of the fundamentals. This objection misunderstands the essence of a model of inattention as described in Sims (2003), since in his theory it does not really matter the amount of information available but how this information is processed by the decisionmakers. In reality, price setters are not even aware about the structure of the model and in any case are in general unsure about the properties and the characteristics of the processes that drive the economy. We interpret the assumption that firms can only observe their own signals as a simple representation of a more complicated world in which there are several dimensions of uncertainty. The gap between the subjective signal and the fundamental shock is a measure of the limitations that firms face in understanding completely the model economy. Overconfidence means that decisionmakers in general over-estimate their ability to understand the correct model.

In our model, decisionmakers are stubborn in their beliefs. Indeed, they do not question at all the structure of the model and treat the parameters as fixed and known. However, they are given a possibility to learn, but a fraction of them optimally decide to remain subjectively informed, given their beliefs. In practice, one reason for why people are overconfident is that there is confirmatory bias (see Soll and Klayman, 2004) - the tendency to interpret the evidence in a way to corroborate their own beliefs. If in our model firms were putting some uncertainty on some parameters of the model or having multiple priors on them, their decision process would have changed substantially from the one detailed in the previous sections and there will be learning from the signal observation. These analyses, although interesting, go beyond the scope of this work and will be subject of further research. 
In this section that goes beyond the framework presented in the previous sections, we are interested to know how quickly an agent outside the model, that treats the parameters of the stochastic process of the signals as fixed but unknown, would detect that firms are overconfident. So think of having one classical statistician per firm that collects period after period the realized signals and that tries to test the beliefs of the firm. In particular, we assume that each statistician is sure that (5.33) and (5.34) are correct and that $\rho=1$ from which it follows

$$
\Delta s_{t}^{j} \equiv s_{t}^{j}-s_{t-1}^{j}=\bar{p}^{*}+u_{t}+\xi_{t}^{j}-\xi_{t-1}^{j}
$$

for each firm $j$. Given the vector of unknown parameters $\phi \equiv\left(\bar{p}^{*}, \sigma_{u}^{2}, \lambda\right)^{\prime}$ and observations $\left\{\Delta s_{t}^{j}\right\}_{t=1}^{T}$ for a sample of size $T$, each statistician can compute the following log-likelihood

$$
\begin{aligned}
\mathcal{L} \mathcal{L}\left(\phi \mid\left\{\Delta s_{t}^{j}\right\}_{t=1}^{T}\right)= & -\frac{T}{2}\left(\ln 2 \pi+\ln \sigma_{u}^{2}\right)-\frac{T}{2} \ln (1 / 2+\lambda+1 / 2 \sqrt{1+4 \lambda}) \\
& -\frac{1}{2 \sigma_{u}^{2}} \frac{1}{(1 / 2+\lambda+1 / 2 \sqrt{1+4 \lambda})} \sum_{t=1}^{T}\left(\Delta s_{t}^{j}-\bar{p}^{*}+\xi_{t-1 \mid t-1}^{j}\right),
\end{aligned}
$$

where the current forecast of $\xi_{t}^{j}$, defined as $\xi_{t \mid t}^{j}$, can be obtained by the following recursive relation

$$
\xi_{t \mid t}^{j}=\frac{\lambda}{(1 / 2+\lambda+1 / 2 \sqrt{1+4 \lambda})}\left(\Delta s_{t}^{j}-\bar{p}+\xi_{t-1 \mid t-1}^{j}\right),
$$

for a given initial condition $\xi_{0 \mid 0}^{j}$.

It is then possible to write the following likelihood ratio to test separately each parameter

$$
\begin{aligned}
& L R_{1}=2\left[\max _{\phi} \mathcal{L} \mathcal{L}\left(\phi \mid\left\{\Delta s_{t}^{j}\right\}_{t=1}^{T}\right)-\max _{\bar{p}^{*}, \sigma_{u}^{2}} \mathcal{L} \mathcal{L}\left(\bar{p}^{*}, \sigma_{u}^{2}, \lambda=\bar{\lambda} \mid\left\{\Delta s_{t}^{j}\right\}_{t=1}^{T}\right)\right] \\
& L R_{2}=2\left[\max _{\phi} \mathcal{L} \mathcal{L}\left(\phi \mid\left\{\Delta s_{t}^{j}\right\}_{t=1}^{T}\right)-\max _{\bar{p}^{*}, \lambda} \mathcal{L} \mathcal{L}\left(\bar{p}^{*}, \sigma_{u}^{2}=\sigma_{u_{0}}^{2}, \lambda \mid\left\{\Delta s_{t}^{j}\right\}_{t=1}^{T}\right)\right] \\
& L R_{3}=2\left[\max _{\phi} \mathcal{L} \mathcal{L}\left(\phi \mid\left\{\Delta s_{t}^{j}\right\}_{t=1}^{T}\right)-\max _{\lambda, \sigma_{u}^{2}} \mathcal{L} \mathcal{L}\left(\bar{p}^{*}=\bar{p}_{0}^{*}, \sigma_{u}^{2}, \lambda \mid\left\{\Delta s_{t}^{j}\right\}_{t=1}^{T}\right)\right]
\end{aligned}
$$

where $\bar{\lambda}, \sigma_{u_{0}}^{2}$, and $\bar{p}_{0}^{*}$ correspond to the values under the respective null. Each of the above test statistics is distributed asymptotically as a $\chi^{2}$ distribution with 1 
degree of freedom. In particular $L R_{1}$ tests whether the belief on $\lambda$, which is equal to $\bar{\lambda}$, is statistically significant; $L R_{2}$ and $L R_{3}$ test $\sigma_{u_{0}}^{2}$ and $\bar{p}_{0}^{*}$, respectively. In the above model, we assumed that the only element of irrationality is the degree of confidence, so $\bar{\lambda}$ does not correspond to the true $\lambda$ while $\sigma_{u_{0}}^{2}$ and $\bar{p}_{0}^{*}$ coincides with the true parameter values.

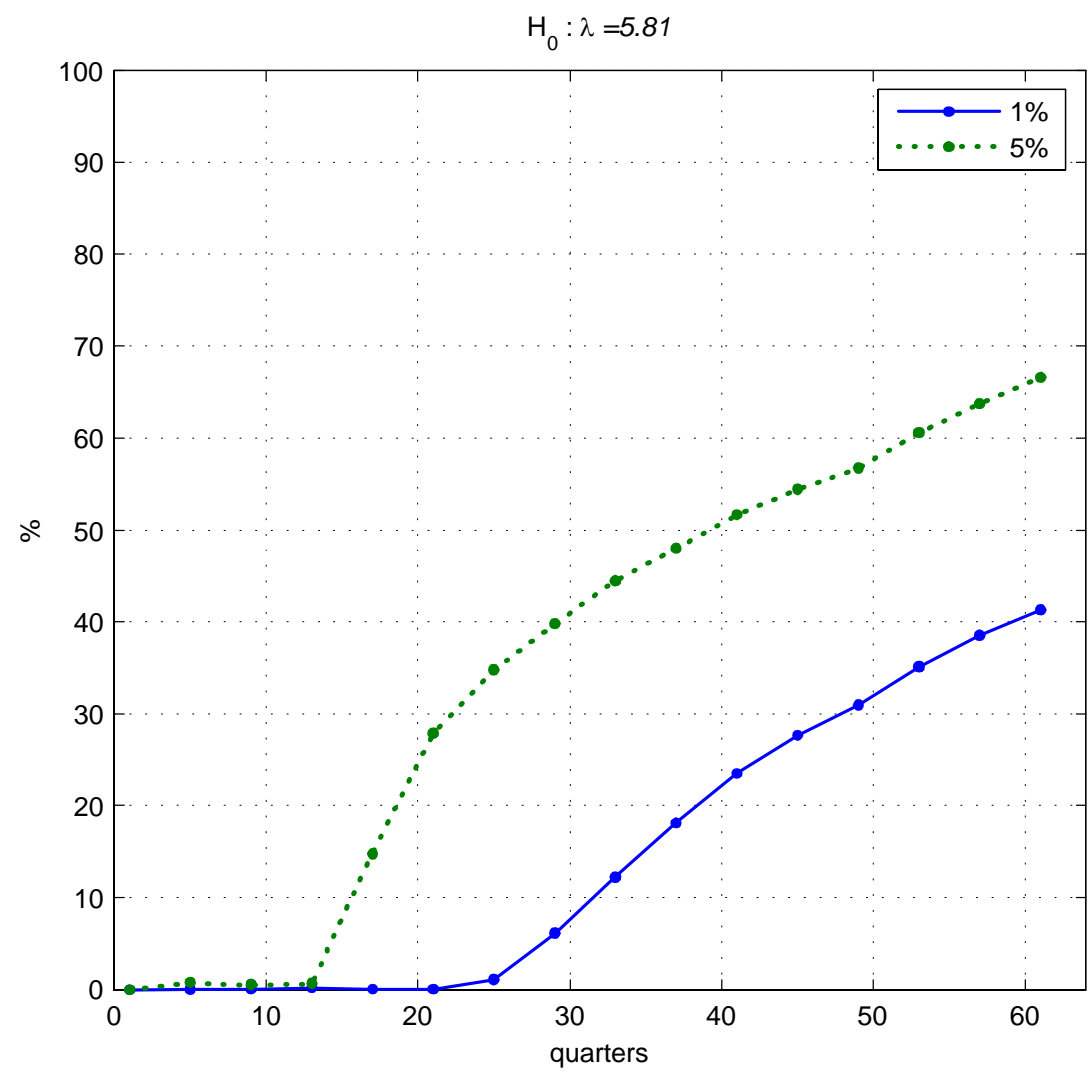

Figure 1: Rejection rate (\%) for the null $\lambda=5.81$ with significance level of $1 \%$ (solid line) and $5 \%$ (dashed line).

Figure 1 (for $L R_{1}$ ), 2 (for $L R_{2}$ ) and 3 (for $L R_{3}$ ) report the share of statisticians that are rejecting the null hypothesis of each test respectively for $T$ that goes from 1 to 61 quarters, as in the KK sample. We run the test for the parameter values that correspond to the calibration when $\eta=0.15$ (first column of Table 1) and for a reasonable degree of overconfidence. In particular $\bar{p}_{0}^{*}=1.11 \%, \sigma_{u_{0}}=4.234 \%$. and $\bar{\lambda}=5.81$, while the true $\lambda$ is 16 times $\bar{\lambda}$. In practice, with the first test we are 


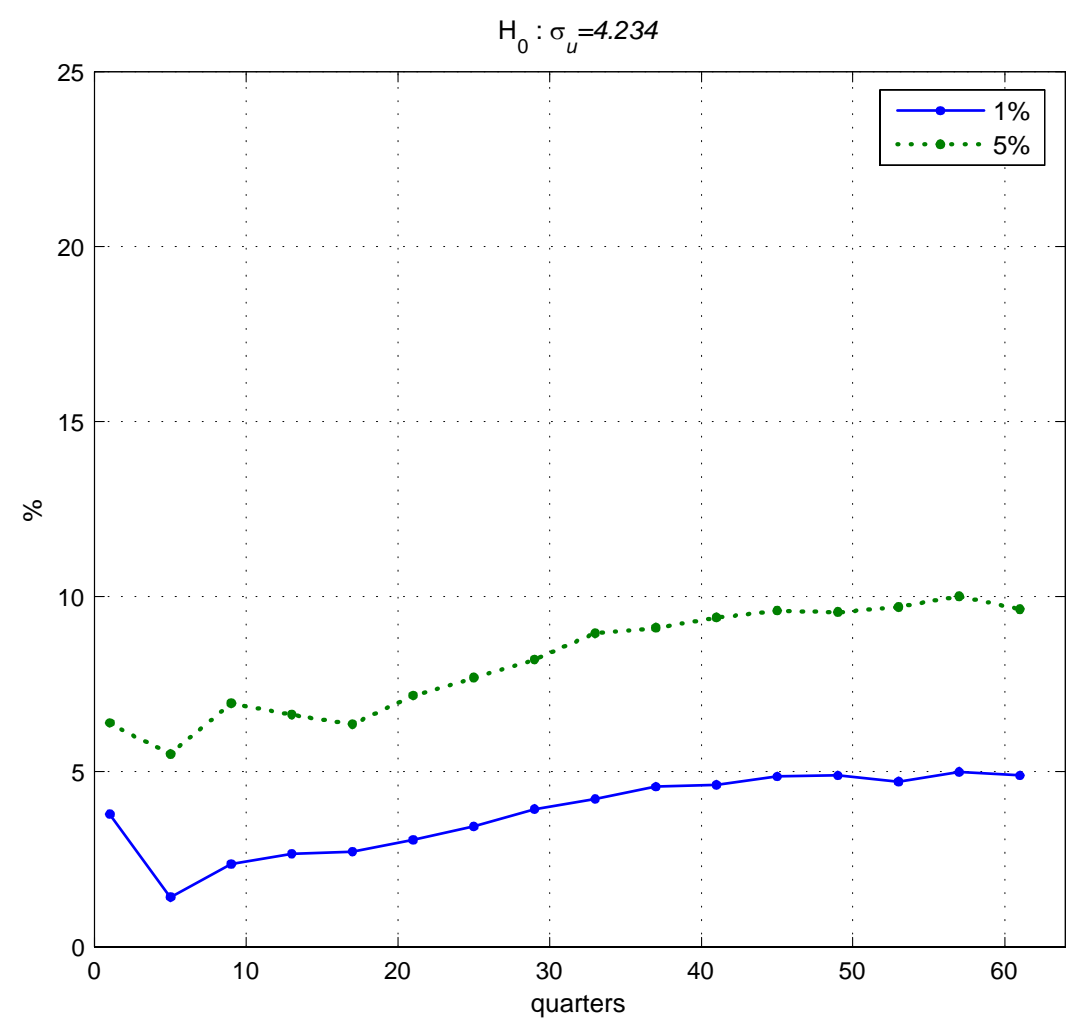

Figure 2: Rejection rate (\%) for the null $\sigma_{u_{0}}=4.234$ with significance level of $1 \%$ (solid line) and 5\% (dashed line).

testing when an index of overconfidence equal to 4 (the $\gamma$ of the previous section) becomes statistically detectable. In each figure, we present results for $1 \%$ and $5 \%$ significance levels. ${ }^{29}$

As expected, the share of statisticians that rejects the null hypothesis in the $\sigma_{u}^{2}$-test and $\bar{p}^{*}$-test is fairly low, since the null corresponds to the truth in these cases. This is shown in Figures 2 and 3 respectively, where the share of rejections is always below $10 \%$ for the $\sigma_{u}^{2}$-test and below $7 \%$ for the $\bar{p}^{*}$-test.

Interestingly enough, it takes a considerable amount of time for the statisticians to detect that the firms are overconfident. We observe in Figure 1 that for a $1 \%$ significance level they start to reject only after the 25th quarter and the share rises

\footnotetext{
${ }^{29}$ We simulated the test 100 times. In each simulation we have 50 firms and the share reported is the average fraction that rejects the null among simulations.
} 


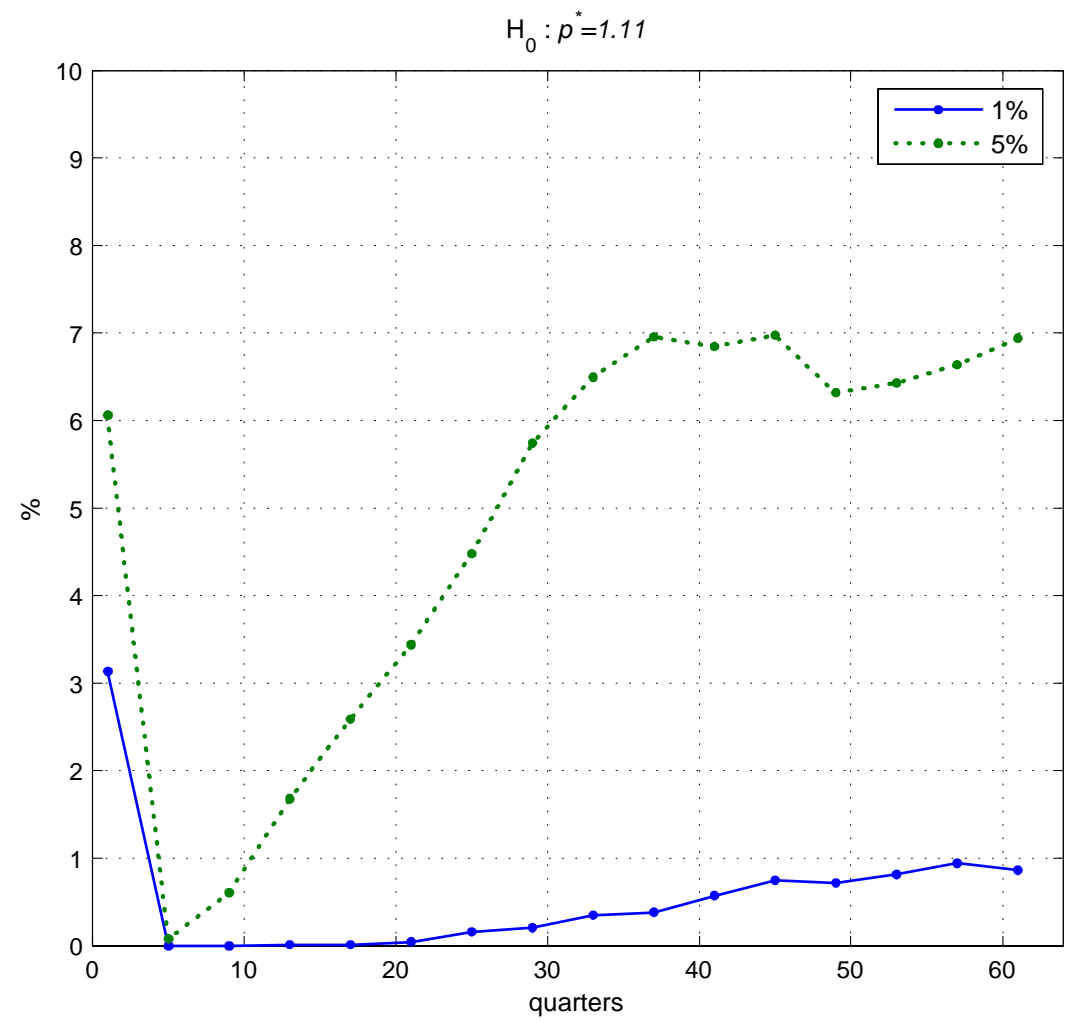

Figure 3: Rejection rate (\%) for the null $\bar{p}^{*}=1.11$ with significance level of $1 \%$ (solid line) and 5\% (dashed line).

only up to $40 \%$ in the 61 st quarter, whereas in the case of the larger critical region which corresponds to a 5\% significance level the share is just $25 \%$ in the 20th quarter and rises to $65 \%$ in the 61st quarter. So despite the considerable amount of overconfidence, the share of statisticians that would detect overconfidence is practically very low.

\section{Conclusion}

In this paper, we have studied the behavior of individual and aggregate prices in a model with monopolistic-competitive firms in an economy that is driven by a hidden state process which is observed with noise. The subjective observation of each firm is not common knowledge to all firms. Moreover, we have assumed that 
this subjective observation is not rational since firms are overconfident and believe that their perceptions are a better representation of the hidden state than they are in reality.

This model can rationalize a persistent response of output to a perturbation to the aggregate hidden state and be consistent at the same time with excess volatility of individual prices since firms pay a lot of attention to their subjective perceptions which include the underestimated noisy component. In our model, firms can in principle choose to discover reality, but their overconfidence prevents them from doing so. This irrational inattention model has then be used to match recent empirical evidence on the behavior of disaggregated prices in the US economy with-

out necessarily assuming high volatility of fundamental aggregate or idiosyncratic shocks.

There are several important assumptions in our stylized framework. Among these is the fact that decisionmakers know (but do not observe) the true structure of the model. What will happen when they doubt which model is going to be unveiled once the noise disappears is the question we leave open for future research.

\section{References}

[1] Allen, Franklin, Stephen Morris and Hyun Song Shin (2005), "Beauty Contests and Iterated Expectations," Review of Financial Studies, forthcoming.

[2] Amato, Jeffery and Hyun Song Shin (2003), "Public and Private Information in Monetary Policy Models," BIS Working Papers No. 138.

[3] Amato, Jeffery and Hyun Song Shin (2006), "Imperfect Common Knowledge and the Information Value of Prices," Economic Theory, 27: 213-241.

[4] Ball, Laurence and David Romer (1989), "Are Prices too Sticky?" The Quarterly Journal of Economics, Vol. 104 No. 3, pp. 507-524.

[5] Ball, Laurence and David Romer (1991), "Sticky Prices as Coordination Failure," American Economic Review, Vol. 81, pp. 539-552.

[6] Battigalli, Pierpaolo (2003), "Game Theory: Games with Asymmetric and Incomplete Information", lecture notes, mimeo, Bocconi University. 
[7] Bils, Mark and Peter J. Klenow (2004), "Some Evidence on the Importance of Sticky Prices," Journal of Political Economy, Vol. 112, pp. 947-985.

[8] Blanchard, Olivier and Nobuhiro Kiyotaki (1987), "Monopolistic Competition and the Effects of Aggregate Demand," The American Economic Review, Vol. 77, No. 4., pp. 647-666.

[9] Compte, Olivier and Andrew Postlewaite (2004), "Confidence-Enhanced Performance," The American Economic Review 94: 1536-1557.

[10] Chari, V. V. and Patrick J. Kehoe, Ellen R. Mcgrattan (2000), "Sticky Price Models of the Business Cycle: Can the Contract Multiplier Solve the Persistence Problem?" Econometrica, Volume: 68, Issue: 5, pp. 1151 - 1179.

[11] Daniel, Kent, David Hirshleifer and Avanidhar Subrahmanyam (2001), "Overconfidence, Arbitrage, and Equilibrium Asset Pricing," The Journal of Finance, Vol. LXI, pp. 921-65.

[12] Daniel, Kent, David Hirshleifer and Siew Hong Teoh (2002), "Investor Psychology in Capital Markets: Evidence and Policy Implications," Journal of Monetary Economics 49: 139-209.

[13] Gertler, Mark and John Leahy (2005), "A Phillips Curve with an Ss Foundation," mimeo, New York University.

[14] Gilboa, Itzhak, and David Schmeidler (1989), "Maximin Expected Utility with Nonunique Prior," Journal of Mathematical Economics 18: 141-153.

[15] Golosov, Mikhail and Robert E. Lucas Jr. (2005), "Menu Costs and Phillips Curves," mimeo, MIT.

[16] Guthrie, C., J.Rachlinski and A. Wistrich (2001), "Inside the Judicial Mind: Heuristics and Biases," Cornell Law Review, Vol.86, pp. 777-830.

[17] Klenow, Peter J. and Oleksiy Kryvtsov (2005), "State-Dependent or TimeDependent Pricing: Does it Matter for Recent U.S Inflation?" mimeo, Stanford University. 
[18] Lucas, Robert E., Jr., (1972), "Expectations and the Neutrality of Money," Journal of Economic Theory 4: 103-124.

[19] Maćkowiak, Bartosz and Mirko Wiederholt (2005), "Optimal Sticky Prices under Rational Inattention," mimeo, Humboldt University, Berlin.

[20] Mankiw, Gregory and Ricardo Reis (2002), "Sticky Information Versus Sticky Prices: A Proposal to Replace the New Keynesian Phillips Curve," Quarterly Journal of Economics, vol. 117 (4), pp. 1295-1328.

[21] Morris, Stephen and Hyun Song Shin (2006), "Inertia of Forward-Looking Expectations," unpublished manuscript, Princeton University.

[22] Oberlechner, Thomas and Carol Osler (2004), "Overconfidence in Currency Markets," unpublished manuscript, University of Vienna.

[23] Phelps, Edmund S. (1970), "Introduction: The New Microeconomics in Employment and Inflation Theory," in E.S. Phelps et al., Microeconomic Foundations of Employment and Inflation Theory, New York: Norton.

[24] Reis, Ricardo (2005), "Inattentive Producers," Review of Economic Studies, forthcoming.

[25] Roll, Richard (1984), "Orange Juice and Weather," American Economic Review 64: 861-880.

[26] Scheinkman, Jose A. and Wei Xiong (2003), "Overconfidence and Speculative Bubbles," Journal of Political Economy, 111: 1183-1220.

[27] Sims, Christopher (2003), "Implications of Rational Inattention," Journal of Monetary Economics, vol. 50, pp. 665-690.

[28] Soll, Jack B. and Klayman, Joshua (2004), "Overconfidence in Interval Estimates," Journal of Experimental Psychology: Learning, Memory, E3 Cognition, 30(2), pp. 299-314.

[29] Svenson, Ola (1981), "Are We All Less Risky and More Skilful than Our Fellow Drivers?" Acta Psychologica, Vol. 47, pp. 143-148. 
[30] Uhlig, Harald (1996), "A Law of Large Numbers for a Large Economy," Economic Theory, vol.8, pp. 41-50.

[31] Woodford, Michael (2002), "Imperfect Common Knowledge and the Effects of Monetary Policy," in P.Aghion, R.Frydman, J.Stiglitz and M.Woodford (eds) Knowledge, Information and Expectations in Modern Macroeconomics: In Honor of Edmund S. Phelps, Princeton University Press: Princeton, pp $25-58$.

[32] Woodford, Michael (2003), Interest and Prices: Foundations of a Theory of Monetary Policy, Princeton University Press: Princeton. 


\section{Appendix}

\section{Derivation of condition (3.12) from (2.11).}

We first note that by using condition (1.7) we can rewrite marginal costs in terms of the complete information price and by using also condition (2.10) we can express the expected profits as functions of $P^{\dagger}(j)$ and $\tilde{P}(j)$ and the variable $Z \equiv M P^{\varepsilon-2}$ as it follows

$$
\begin{gathered}
E^{j}\left\{\pi\left(P^{\dagger}(j), P, \theta\right)\right\}=\frac{1}{\varepsilon} E^{j}\left\{\left(P^{\dagger}(j)\right)^{1-\varepsilon} Z\right\}, \\
E^{j}\{\pi(\tilde{P}(j), P, \theta)\}=\frac{1}{\varepsilon} \tilde{P}^{1-\varepsilon}(j) E^{j} Z .
\end{gathered}
$$

We take a second order approximation of expected profits around a deterministic steady state where $\theta=\bar{\theta}$ and as a result $P=P^{\dagger}=\tilde{P} \equiv \bar{P}$. Let lowercase variables denote log-deviations from the steady state and let $\|p\|$ and $\|z\|$ denote a bound on the size of fluctuations for the price of each differentiated good and for the variable $Z$ respectively. We can obtain by approximating equation (A.1) that

$$
\begin{aligned}
E^{j}\left\{\pi\left(P^{\dagger}(j), P, \theta\right)\right\}= & \frac{1}{\varepsilon} \bar{P}^{1-\varepsilon} \bar{Z}\left[1+(1-\varepsilon) E^{j} p^{\dagger}+\frac{1}{2}(1-\varepsilon)^{2} E^{j}\left(p^{\dagger}\right)^{2}+\right. \\
& \left.+E^{j} z+\frac{1}{2} E^{j} z^{2}+(1-\varepsilon) E^{j} p^{\dagger} z\right]+\mathcal{O}\left(\|p, z\|^{3}\right) .
\end{aligned}
$$

Similarly by approximating equation (A.2) we obtain that

$$
\begin{aligned}
E^{j}\{\pi(\tilde{P}(j), P, \theta)\}= & \frac{1}{\varepsilon} \bar{P}^{1-\varepsilon} \bar{Z}\left[1+(1-\varepsilon) \tilde{p}(j)+\frac{1}{2}(1-\varepsilon)^{2} \tilde{p}(j)^{2}\right. \\
& \left.+E^{j} z+\frac{1}{2} E^{j} z^{2}+(1-\varepsilon) \tilde{p}(j) E^{j} z\right]+\mathcal{O}\left(\|p, z\|^{3}\right) \cdot(
\end{aligned}
$$

Note that $\bar{P}^{1-\varepsilon} \bar{Z}=\frac{\bar{M}}{P}=\bar{Y}$ and let

$$
W(j) \equiv \pi\left(P^{\dagger}(j), P, \theta\right)-\pi(\tilde{P}(j), P, \theta)
$$

denote the difference in profits. Then using (A.3) and (A.4) we have

$$
\begin{aligned}
E^{j}\{W(j)\}= & \frac{\varepsilon-1}{\varepsilon} \bar{Y}\left\{\tilde{p}(j)-E^{j} p^{\dagger}+\frac{1}{2}(\varepsilon-1)\left[E^{j}\left(p^{\dagger}\right)^{2}-(\tilde{p}(j))^{2}\right]\right. \\
& \left.-E^{j}\left[\left(p^{\dagger}-\tilde{p}(j)\right) z\right]\right\}+\mathcal{O}\left(\|p, z\|^{3}\right) .
\end{aligned}
$$


Note that the price of the uninformed agents (1.1) in a first order approximation is

$$
\tilde{p}(j)=E^{j} p^{\dagger}+\mathcal{O}\left(\|p, z\|^{2}\right) .
$$

Furthermore, if we take a second-order approximation of it we obtain

$$
\tilde{p}(j)-E^{j} p^{\dagger}=\frac{1}{2} \operatorname{Var}^{j}\left(p^{\dagger}\right)+E^{j}\left[p^{\dagger}-E^{j} p^{\dagger}\right] z+\mathcal{O}\left(\|p, z\|^{3}\right),
$$

where $\operatorname{Var}^{j}\left(p^{\dagger}\right) \equiv E^{j}\left(p^{\dagger}\right)^{2}-\left(E^{j} p^{\dagger}\right)^{2}$. Using (A.6) and (A.7) into (A.5) we observe that the terms involving $z$ cancel out and that $E^{j}\left(p^{\dagger 2}-\tilde{p}(j)\right)^{2}=\operatorname{Var}^{j}\left(p^{\dagger}\right)$, so ignoring third order terms we obtain

$$
\begin{aligned}
E^{j}\{W(j)\} & =\frac{1}{2} \frac{\varepsilon-1}{\varepsilon} \bar{Y}\left\{\operatorname{Var}^{j}\left(p^{\dagger}\right)+(\varepsilon-1) \operatorname{Var}^{j}\left(p^{\dagger}\right)\right\} \\
& =\frac{\bar{Y}}{2}(\varepsilon-1) \operatorname{Var}^{j}\left(p^{\dagger}\right) .
\end{aligned}
$$

Thus firms acquire information if and only if

$$
\operatorname{Var}^{j}\left(p^{\dagger}\right) \geq c^{j}
$$

where $c^{j} \equiv \frac{2}{Y(\varepsilon-1)} \tilde{c}^{j}$ which is expression (3.12) in the text. In the text, we also denote with lower-case letters the logs of the respective variable.

Note that exactly the same calculations apply in the dynamic case, where each variable is indexed with $t$. In this case the expected difference of profits at time $t$, in $(5.31)$, is equal to

$$
E_{t}^{j}\left\{\pi\left(P_{t}^{\dagger}(j), P_{t}, \theta_{t}\right)-\pi\left(\tilde{P}_{t}(j), P_{t}, \theta_{t}\right)\right\}=\frac{\bar{Y}}{2}(\varepsilon-1) \operatorname{Var}_{t}^{j}\left(p_{t}^{\dagger}\right)
$$

in a second-order approximation, where the expectation operator is conditional on the private history of signals including time $t$. So $\operatorname{Var}_{t}^{j}\left(p_{t}^{\dagger}\right)$ corresponds to the subjective contemporaneous variance of $p_{t}^{\dagger}$.

\section{Derivations of (5.35) and (5.36).}

We proceed using the method developed in Woodford (2002). We claim that the relevant hidden state is

$$
X_{t}=\left[\begin{array}{c}
p_{t}^{*} \\
p_{t}
\end{array}\right]
$$


and guess that it evolves according to a linear law of motion

$$
X_{t}=f+M X_{t-1}+m u_{t},
$$

where

$$
f \equiv\left[\begin{array}{c}
\bar{p}^{*} \\
\bar{p}
\end{array}\right], \quad M \equiv\left[\begin{array}{cc}
\rho & 0 \\
a & b
\end{array}\right], \quad m \equiv\left[\begin{array}{l}
1 \\
c
\end{array}\right],
$$

are vectors and matrices to be determined. Note that our variables of interest are the prices of the informed firms which can be written as $p^{\dagger}=\bar{\eta}^{\prime} X_{t}$ and that of the uninformed which can be written as $\tilde{p}_{t}(i)=\bar{\eta}^{\prime} E_{t}^{i} X_{t}$, where $\bar{\eta}^{\prime}=(\eta, 1-\eta)$.

Let $e_{1}=(1,0)^{\prime}$. We can write the following system

$$
\begin{aligned}
X_{t} & =f+M X_{t-1}+m u_{t} \\
s_{t}^{i} & =e_{1}^{\prime} X_{t}+\xi_{t}^{j}
\end{aligned}
$$

where the second line corresponds to the observational equation. We proceed assuming a stationary filtering problem. The filtering equation of a generic uninformed firm $j$ is given by

$$
E_{t}^{j} X_{t}=E_{t-1}^{j} X_{t}+K\left(s_{t}^{j}-E_{t-1}^{j} p_{t}^{*}\right),
$$

where $K$ is the vector of Kalman gains pre-multiplied with $M^{-1}$. Using (A.8) we obtain that $E_{t-1}^{j} X_{t}=f+M E_{t-1}^{j} X_{t-1}$ and $E_{t-1}^{j} p_{t}^{*}=\bar{p}^{*}+\rho E_{t-1}^{j} p_{t-1}^{*}$; we can then write (A.9) as

$$
E_{t}^{j} X_{t}=f+M E_{t-1}^{j} X_{t-1}+K\left(s_{t}^{j}-\bar{p}^{*}-\rho E_{t-1}^{j} p_{t-1}^{*}\right) .
$$

Aggregating among all agents $j$ that are uninformed and guessing that in equilibrium $\mu$ will be non-random (as in the static case) we obtain

$$
\begin{aligned}
\bar{E}_{t} X_{t} & =f+M \bar{E}_{t-1} X_{t-1}+K\left(p_{t}^{*}-\bar{p}^{*}-\rho \bar{E}_{t-1} p_{t-1}^{*}\right), \\
& =f+M \bar{E}_{t-1} X_{t-1}+\rho K\left(p_{t-1}^{*}-\bar{E}_{t-1} p_{t-1}^{*}\right)+K u_{t},
\end{aligned}
$$

which is the law of motion of the average estimate, where we have used the law of large numbers.

Our target is to express the price level $p_{t}$ in terms of $X_{t-1}$. The general price index can be expressed as a function of the full information price and average 
expectations as

$$
\begin{aligned}
p_{t} & =\delta\left[(1-\mu) p_{t}^{*}+\mu \bar{E}_{t} p_{t}^{*}\right]+(1-\delta) \bar{E}_{t} p_{t} \\
& =(\delta \mu, 1-\delta) \bar{E}_{t} X_{t}+\delta(1-\mu) p_{t}^{*} \\
& =(\delta \mu, 1-\delta) \bar{E}_{t} X_{t}+\delta(1-\mu)\left(\bar{p}^{*}+\rho p_{t-1}^{*}+u_{t}\right)
\end{aligned}
$$

Using the law of motion (A.10) to substitute for $\bar{E}_{t} X_{t}$ and collecting terms we have

$$
\begin{aligned}
p_{t}= & {\left[(\delta \mu, 1-\delta) f+\delta(1-\mu) \bar{p}^{*}\right]+\rho[\delta(1-\mu)+\bar{K}] p_{t-1}^{*}+(\delta \mu, 1-\delta) M \bar{E}_{t-1} X_{t-1} } \\
& -\rho \bar{K} \bar{E}_{t-1} p_{t-1}^{*}+[\delta(1-\mu)+\bar{K}] u_{t},
\end{aligned}
$$

where $\bar{K} \equiv(\delta \mu, 1-\delta) K$. Finally, using the definition of $M$ and $\bar{E}_{t-1} X_{t-1}$ and noting that

$$
(\delta \mu, 1-\delta) f+\delta(1-\mu) \bar{p}^{*}=\delta \bar{p}^{*}+(1-\delta) \bar{p}
$$

we obtain that

$$
\begin{aligned}
p_{t}= & \delta \bar{p}^{*}+(1-\delta) \bar{p}+\rho[\delta(1-\mu)+\bar{K}] p_{t-1}^{*}+[\delta \mu \rho+(1-\delta) a-\rho \bar{K}] \bar{E}_{t-1} p_{t-1}^{*} \\
& +(1-\delta) b \bar{E}_{t-1} p_{t-1}+[\delta(1-\mu)+\bar{K}] u_{t} .
\end{aligned}
$$

Since

$$
p_{t-1}=\delta \mu \bar{E}_{t-1} p_{t-1}^{*}+(1-\delta) \bar{E}_{t-1} p_{t-1}+\delta(1-\mu) p_{t-1}^{*},
$$

we can use this expression to substitute for $\bar{E}_{t-1} p_{t-1}$ in (A.11) and arrive at

$$
\begin{aligned}
p_{t}= & \delta \bar{p}^{*}+(1-\delta) \bar{p}+[\delta(1-\mu)(\rho-b)+\rho \bar{K}] p_{t-1}^{*} \\
& +[\delta \mu(\rho-b)+(1-\delta) a-\rho \bar{K}] \bar{E}_{t-1} p_{t-1}^{*} \\
& +b p_{t-1}+[\delta(1-\mu)+\bar{K}] u_{t}
\end{aligned}
$$

We note that (A.8) implies

$$
p_{t}=\bar{p}+a p_{t-1}^{*}+b p_{t-1}+c u_{t}
$$

We can then match the coefficients between (A.12) and (A.13) and obtain

$$
\begin{aligned}
\delta \bar{p}^{*}+(1-\delta) \bar{p} & =\bar{p} \\
\delta(1-\mu)(\rho-b)+\rho \bar{K} & =a \\
\delta \mu(\rho-b)+(1-\delta) a-\rho \bar{K} & =0 \\
\delta(1-\mu)+\bar{K} & =c .
\end{aligned}
$$


Solving this system and using the definition of $\bar{K}=(\delta \mu, 1-\delta) K$, we get

$$
\begin{aligned}
\bar{p} & =\bar{p}^{*} \\
a & =\frac{\rho}{\delta \mu+1-\delta} \bar{K}=\rho \bar{\eta}^{\prime} K=\rho \hat{k}
\end{aligned}
$$

since $\eta=\delta \mu /(\delta \mu+1-\delta)$ and $\hat{k} \equiv \bar{\eta}^{\prime} K$. Moreover

$$
b=\rho-a=\rho(1-\hat{k})
$$

and

$$
c=(\delta \mu+1-\delta) \hat{k}+\delta(1-\mu) .
$$

The vector of (pre-multiplied) Kalman gains satisfies the equation

$$
K=\Sigma e_{1}\left(e_{1}^{\prime} \Sigma e_{1}+\bar{\sigma}_{\xi}^{2}\right)^{-1},
$$

where $\Sigma$ is the variance of the one step ahead forecast error which satisfies the following stationary version of the Riccatti equation

$$
\Sigma=M \Sigma M^{\prime}+m m^{\prime} \sigma_{u}^{2}-\left(e_{1}^{\prime} \Sigma e_{1}+\bar{\sigma}_{\xi}^{2}\right)^{-1} M \Sigma e_{1} e_{1}^{\prime} \Sigma M^{\prime} .
$$

Thus in our guess-and-verify approach we expressed $M$ and $m$ as a function of $\hat{k}$ which depends on the vector of Kalman gains $K$ which in turn depends on $\Sigma$. But $\Sigma$ depends on $M$ and $m$ by (A.15). So it remains to solve for this fixed point. Let

$$
\Sigma=\left[\begin{array}{ll}
\sigma_{11} & \sigma_{12} \\
\sigma_{12} & \sigma_{22}
\end{array}\right]
$$

Solving the upper left block of the Riccatti equation (A.15) we find that $\sigma_{11}$ satisfies the quadratic

$$
\sigma_{11}^{2}+\left[\left(1-\rho^{2}\right) \bar{\sigma}_{\xi}^{2}-\sigma_{u}^{2}\right] \sigma_{11}-\bar{\sigma}_{\xi}^{2} \sigma_{u}^{2}=0 .
$$

The positive root (since $\sigma_{11}$ is a variance) of this quadratic is

$$
\sigma_{11}=\frac{1}{2} \sigma_{u}^{2}\left\{1-\left(1-\rho^{2}\right) \bar{\lambda}+\sqrt{\left(1-\left(1-\rho^{2}\right) \bar{\lambda}\right)^{2}+4 \bar{\lambda}}\right\}
$$

where $\bar{\lambda} \equiv \frac{\bar{\sigma}_{\xi}^{2}}{\sigma_{u}^{2}}$. From the lower left block of the Riccatti we derive

$$
\sigma_{12}=\frac{\rho^{2} \hat{k} \sigma_{11} \bar{\sigma}_{\xi}^{2}+\left(\sigma_{11}+\bar{\sigma}_{\xi}^{2}\right)\left[(\delta \mu+1-\delta) \hat{k} \sigma_{u}^{2}+\delta(1-\mu) \sigma_{u}^{2}\right]}{\left[1-\rho^{2}(1-\hat{k})\right] \bar{\sigma}_{\xi}^{2}+\sigma_{11}}
$$


and -using (A.14)- we obtain

$$
\hat{k}=\bar{\eta}^{\prime} K=\eta \frac{\sigma_{11}}{\sigma_{11}+\bar{\sigma}_{\xi}^{2}}+(1-\eta) \frac{\sigma_{12}}{\sigma_{11}+\bar{\sigma}_{\xi}^{2}}
$$

This is a system of two equations in the two unknowns $\left(\sigma_{12}, \hat{k}\right)$. Solving the system and using our solution for $\sigma_{11}$ we finally arrive at the quadratic expression for $\hat{k}$

$$
Q(\hat{k})=\rho^{2} \bar{\lambda} \hat{k}^{2}+\left[\bar{\lambda}\left(1-\rho^{2}\right)+\delta\right] \hat{k}-\delta=0 .
$$

The discriminant of (A.16) is positive, so there are two real roots. Furthermore, since $Q(0)<0$ and $Q(1)=\bar{\lambda}>0$, one is negative and the other positive and less than unity. Note that subtracting $p_{t}$ from $p_{t}^{*}$ we get an expression for the output deviation

$$
y_{t}=\rho(1-\hat{k}) y_{t-1}+(1-c) u_{t}
$$

since $p_{t}^{*}+\ln \bar{Y}=\ln M_{t}=p_{t}+\ln Y_{t}$. In order to have a stationary solution for output we need $|1-\hat{k}|<|\rho|^{-1}$. It follows that $\hat{k}$ should satisfy the restriction $1-\rho^{-1}<\hat{k}<1+\rho^{-1}$. Since $Q\left(1-\rho^{-1}\right)=-\rho^{-1}\left((\rho-1)^{2} \lambda+\delta\right)<0$, only the positive root of the quadratic satisfies the restriction. Thus

$$
\hat{k}=\frac{1}{2 \rho^{2}}\left\{\rho^{2}-1-\frac{\delta}{\bar{\lambda}}+\sqrt{\left[\left(1-\rho^{2}\right)+\frac{\delta}{\bar{\lambda}}\right]^{2}+4 \rho^{2} \frac{\delta}{\bar{\lambda}}}\right\} .
$$

Having solved for the laws of motion of $X_{t}$ and $E_{t}^{j} X_{t}$, we can derive the laws of motion of the prices of interest $p^{\dagger}=\bar{\eta}^{\prime} X_{t}$ and $\tilde{p}_{t}(j)=\bar{\eta}^{\prime} E_{t}^{j} X_{t}$ and obtain expressions (5.35) and (5.36) in the main text, respectively.

\section{Derivations of (5.37).}

Defining $q_{t}^{j} \equiv p_{t}^{\dagger}-\tilde{p}_{t}(j)=p_{t}^{\dagger}-E_{t}^{j} p_{t}^{\dagger}$ we obtain

$$
q_{t}^{j}=\rho(1-\hat{k}) q_{t-1}^{j}+\delta(1-\hat{k}) u_{t}-\hat{k} \xi_{t}^{j}
$$

At first notice that $E_{t}^{j} q_{t}^{j}=E_{t}^{j}\left(p_{t}^{\dagger}-\tilde{p}_{t}(j)\right)=0$ and $E_{t-1}^{j} q_{t}^{j}=E_{t-1}^{j}\left(p_{t}^{\dagger}-\tilde{p}_{t}(j)\right)=$ $E_{t-1}^{j} p_{t}^{\dagger}-E_{t-1}^{j} E_{t}^{j} p_{t}^{\dagger}=0$. Calculating variances conditional on the private history until last period we get

$$
\operatorname{Var}_{t-1}^{j}\left(q_{t}^{j}\right)=\rho^{2}(1-\hat{k})^{2} \operatorname{Var}_{t-1}^{j}\left(q_{t-1}^{j}\right)+\delta^{2}(1-\hat{k})^{2} \sigma_{u}^{2}+\hat{k}^{2} \bar{\sigma}_{\xi}^{2} .
$$


Note that $\operatorname{Var}_{t-1}^{j}\left(q_{t-1}^{j}\right)=E_{t-1}^{j}\left(q_{t-1}^{j}\right)^{2}=\operatorname{Var}_{t-1}^{j}\left(p_{t-1}^{\dagger}\right)$. Moreover, $\operatorname{Var}_{t-1}^{j}\left(q_{t}^{j}\right)=$ $E_{t-1}^{j}\left(q_{t}^{j}\right)^{2}=E_{t-1}^{j}\left\{E_{t}^{j}\left(q_{t}^{j}\right)^{2}\right\}=E_{t-1}^{j} \operatorname{Var}_{t}^{j}\left(p_{t}^{\dagger}\right)=\operatorname{Var}_{t}^{j}\left(p_{t}^{\dagger}\right)$, where the last step follows from the non-randomness of the variances of the filter. The expression in the text for the contemporaneous variance follows by using the stationarity of the filter and the fact that (A.16) implies $\delta(1-\hat{k})=\bar{\lambda} \hat{k}\left[1-\rho^{2}(1-\hat{k})\right]$.

\section{Derivations of (5.39).}

We will now proceed to derive the condition for excess volatility of the prices of the uninformed firms. Taking unconditional variances in (A.17) we obtain that

$$
\operatorname{var}\left(q_{t}^{j}\right)=\frac{1}{1-\rho^{2}(1-\hat{k})^{2}}\left[\delta^{2}(1-\hat{k})^{2} \sigma_{u}^{2}+\hat{k}^{2} \sigma_{\xi}^{2}\right] .
$$

Furthermore note that, since

$$
\operatorname{var}\left(q_{t}^{j}\right)=\operatorname{var}\left(p_{t}^{\dagger}\right)+\operatorname{var}\left(\tilde{p}_{t}(j)\right)-2 \operatorname{cov}\left(p_{t}^{\dagger}, \tilde{p}_{t}(j)\right)
$$

and

$$
\operatorname{cov}\left(p_{t}^{\dagger}, \tilde{p}_{t}(j)\right)=\operatorname{cov}\left(q_{t}^{j}, \tilde{p}_{t}(j)\right)+\operatorname{var}\left(\tilde{p}_{t}(j)\right),
$$

we have

$$
\operatorname{var}\left(p_{t}^{\dagger}\right)=\operatorname{var}\left(q_{t}^{j}\right)+2 \operatorname{cov}\left(q_{t}^{j}, \tilde{p}_{t}(j)\right)+\operatorname{var}\left(\tilde{p}_{t}(j)\right) .
$$

Dividing over $\operatorname{var}\left(p_{t}^{\dagger}\right)$ we obtain

$$
\frac{\operatorname{var}\left(\tilde{p}_{t}(j)\right)}{\operatorname{var}\left(p_{t}^{\dagger}\right)}=1-\frac{\operatorname{var}\left(q_{t}^{j}\right)+2 \operatorname{cov}\left(q_{t}^{j}, \tilde{p}_{t}(j)\right)}{\operatorname{var}\left(p_{t}^{\dagger}\right)} .
$$

So the ratio can exceed unity only if $I \equiv \operatorname{var}\left(q_{t}^{j}\right)+2 \operatorname{cov}\left(q_{t}^{j}, \tilde{p}_{t}(j)\right)<0$. Note that $\operatorname{cov}\left(q_{t}^{j}, \tilde{p}_{t}(j)\right)=\frac{1}{1-\rho^{2}(1-\hat{k})^{2}}\left[\rho^{2}(1-\hat{k}) \hat{k} \cdot \operatorname{cov}\left(p_{t-1}^{*}, q_{t-1}^{j}\right)+\delta(1-\hat{k}) \hat{k} \sigma_{u}^{2}-\hat{k}^{2} \sigma_{\xi}^{2}\right]$.

Using the law of motion for the full information price and $q_{t}^{j}$ we derive that

$$
\operatorname{cov}\left(p_{t}^{*}, q_{t}^{j}\right)=\frac{\delta(1-\hat{k}) \sigma_{u}^{2}}{1-\rho^{2}(1-\hat{k})}
$$

and plugging it in the previous expression we finally obtain

$$
\operatorname{cov}\left(q_{t}^{j}, \tilde{p}_{t}(j)\right)=\frac{1}{1-\rho^{2}(1-\hat{k})^{2}}\left[\frac{\delta(1-\hat{k}) \hat{k}}{1-\rho^{2}(1-\hat{k})} \sigma_{u}^{2}-\hat{k}^{2} \sigma_{\xi}^{2}\right] .
$$


Therefore

$$
I=\frac{\hat{k}^{2}}{1-\rho^{2}(1-\hat{k})^{2}}\left[\frac{\delta(1-\hat{k})}{\hat{k}}\left(\frac{\delta(1-\hat{k})}{\hat{k}}+\frac{2}{1-\rho^{2}(1-\hat{k})}\right)-\lambda\right] \sigma_{u}^{2} .
$$

In order to have $I<0$, we need

$$
\frac{\delta(1-\hat{k})}{\hat{k}}\left[\frac{\delta(1-\hat{k})}{\hat{k}}+\frac{2}{1-\rho^{2}(1-\hat{k})}\right]-\lambda<0 .
$$

Using as before the fact that $\delta(1-\hat{k})=\bar{\lambda} \hat{k}\left[1-\rho^{2}(1-\hat{k})\right]$ we derive the condition (5.39).

\section{Calibration of the information costs.}

We convert the lifetime costs to costs per period by multiplying it by the factor $(1-\beta)$. The steady state real profits are $\bar{\pi}=\bar{Y} / \varepsilon$. Recall that

$$
c^{j}=\frac{2}{(\varepsilon-1)} \frac{\tilde{c}^{j}}{\bar{Y}}
$$

Then the equilibrium fraction of uninformed agents is given by

$$
\mu=1-\operatorname{Fr}\left(c^{j} \leq c^{*}\right)
$$

where $\operatorname{Fr}\left(c^{j} \leq c^{*}\right)$ measures the frequency (or the mass) of costs below $c^{*}$. In particular

$$
\operatorname{Fr}\left(c^{j} \leq c^{*}\right)=\operatorname{Fr}\left(c^{j}(1-\beta) \leq c^{*}(1-\beta)=\operatorname{Var}_{t}^{j}\left(p_{t}^{\dagger}\right)\right)
$$

and

$$
\operatorname{Fr}\left(\frac{\tilde{c}^{j}(1-\beta)}{\bar{Y}} \leq \frac{(\varepsilon-1)}{2} \operatorname{Var}_{t}^{j}\left(p_{t}^{\dagger}\right)\right)=\operatorname{Fr}\left(\frac{\tilde{c}^{j}(1-\beta)}{\bar{\pi}} \leq \frac{\varepsilon(\varepsilon-1)}{2} \operatorname{Var}_{t}^{j}\left(p_{t}^{\dagger}\right)\right) .
$$

Given our assumption of a uniform distribution for the costs as a fraction of steady state real profits with minimum zero and mean $4.61 \%$, it follows that

$$
\mu=1-U\left(\frac{\varepsilon(\varepsilon-1)}{2} \operatorname{Var}_{t}^{j}\left(p_{t}^{\dagger}\right)\right)
$$

where $U(\cdot)$ is the corresponding c.d.f. This equation gives the implicit equation (6.42). 